\title{
Augmented antibody-based anticancer therapeutics boost neutrophil cytotoxicity
}

\author{
Niels Heemskerk, ${ }^{1}$ Mandy Gruijs, ${ }^{1}$ A. Robin Temming, ${ }^{2}$ Marieke H. Heineke, ${ }^{1}$ Dennis Y. Gout, ${ }^{1}$ Tessa Hellingman, ${ }^{3}$ \\ Cornelis W. Tuk, ${ }^{1}$ Paula J. Winter, ${ }^{1}$ Suzanne Lissenberg-Thunnissen, ${ }^{2}$ Arthur E.H. Bentlage, ${ }^{2}$ Marco de Donatis, ${ }^{1}$ Marijn Bögels, ${ }^{1}$ \\ Thies Rösner, ${ }^{4}$ Thomas Valerius, ${ }^{4}$ Jantine E. Bakema, ${ }^{5}$ Gestur Vidarsson, ${ }^{2}$ and Marjolein van Egmond ${ }^{1,3}$ \\ 1'Amsterdam UMC, Vrije Universiteit Amsterdam, Department of Molecular Cell Biology and Immunology, Cancer Center Amsterdam, Amsterdam Infection and Immunity, De Boelelaan 1117, Amsterdam, \\ Netherlands. ${ }^{2}$ Sanquin Research and Landsteiner Laboratory, Department of Experimental Immunohematology, Amsterdam UMC, University of Amsterdam, Amsterdam, Netherlands. ${ }^{3}$ Amsterdam UMC, \\ Vrije Universiteit Amsterdam, Department of Surgery, Cancer Center Amsterdam, De Boelelaan 1117, Amsterdam, Netherlands. ${ }^{4}$ Section for Stem Cell Transplantation and Immunotherapy, Department of \\ Medicine II, Christian Albrechts University and University Hospital Schleswig Holstein, Kiel, Germany. ${ }^{5}$ Amsterdam UMC, Vrije Universiteit Amsterdam, Department of Otolaryngology/Head-Neck Surgery, \\ Cancer Center Amsterdam, De Boelelaan 1117, Amsterdam, Netherlands.
}

\begin{abstract}
Most clinically used anticancer mAbs are of the IgG isotype, which can eliminate tumor cells through NK cell-mediated antibody-dependent cellular cytotoxicity and macrophage-mediated antibody-dependent phagocytosis. IgG, however, ineffectively recruits neutrophils as effector cells. IgA mAbs induce migration and activation of neutrophils through the lgA Fc receptor (FcaRI) but are unable to activate NK cells and have poorer half-life. Here, we combined the agonistic activity of IgG mAbs and FcaRI targeting in a therapeutic bispecific antibody format. The resulting TrisomAb molecules recruited NK cells, macrophages, and neutrophils as effector cells for eradication of tumor cells in vitro and in vivo. Moreover, TrisomAb had long in vivo half-life and strongly decreased B16F10gp75 tumor outgrowth in mice. Importantly, neutrophils of colorectal cancer patients effectively eliminated tumor cells in the presence of anti-EGFR TrisomAb but were less efficient in mediating killing in the presence of IgG anti-EGFR mAb (cetuximab). The clinical application of TrisomAb may provide potential alternatives for cancer patients who do not benefit from current IgG mAb therapy.
\end{abstract}

\section{Introduction}

Immunotherapy is a promising addition to classical cancer treatments like surgery, chemotherapy, and radiotherapy (1-4). Treatment with mAbs that target hematological malignancies, such as anti-CD20 ( $\alpha$-CD20) mAbs, can effectively and rapidly eliminate malignant cells, although not all patients achieve complete remission and others experience relapse $(5,6)$. mAbs are also used to treat solid malignancies. For instance, $\alpha$-EGFR mAbs are used for the treatment of metastasized colorectal and head and neck cancer (7). However, compared with the success of treating hematological malignancies, results in solid cancers are modest $(8,9)$. Improvement of antibody-based immunotherapeutic approaches is therefore urgently needed.

Most mAbs that are used in the clinic are of the IgG1 isotype and act through multiple mechanisms. Antitumor IgG1 mAbs can exert their antitumor activity by blocking growth factor receptors or apoptosis induction $(2,10)$. Additionally, they can activate the classical complement pathway (11) and recruit innate effector cells through binding to $\mathrm{Fc}$ gamma receptors (Fc $\gamma \mathrm{Rs}$ ). Both NK cells and macrophages represent potent effector cells in IgG1-mediated tumor cell

Authorship note: MC and ART contributed equally to this work. Conflict of interest: The authors have declared that no conflict of interest exists. Copyright: (๖) 2021, American Society for Clinical Investigation.

Submitted: November 1, 2019; Accepted: February 3, 2021; Published: February 9, 2021. Reference information: J Clin Invest. 2021;131(6):e134680.

https://doi.org/10.1172/JCI134680. killing. NK cells induce apoptosis via release of perforins and granzymes or through activation of death receptor pathways, which is referred to as antibody-dependent cellular cytotoxicity (ADCC) (12, 13). Macrophages eliminate antibody-opsonized tumor cells mainly through antibody-dependent cellular phagocytosis (ADCP) $(14,15)$. It has been shown that Fc $\gamma$ R-mediated mechanisms are essential for therapeutic antitumor efficacy in several mouse models (16-18). Furthermore, Fc $\gamma \mathrm{R}$ polymorphisms that influence binding affinity for IgG correlated to some extent with clinical outcome after treatment with rituximab ( $\alpha$-CD20) or cetuximab ( $\alpha$-EGFR) in lymphoma or colorectal cancer, respectively (19-21), suggesting that ADCC or ADCP may play a role. Nonetheless, mutations in EGFR signaling pathways (e.g., KRAS) have a bigger impact on therapeutic efficacy $(22,23)$, supporting the notion that enlisting NK cells and macrophages as effector cells in mAb therapy is not sufficient.

Neutrophils, which represent the largest cytotoxic cell population in the circulation, have previously been proposed as an additional effector cell population in mAb therapy of cancer $(24,25)$. Via cotreatment with G-CSF, their number can be easily expanded without the need for complicated ex vivo culture or manipulation (26). Neutrophils furthermore kill tumor cells via several nonapoptotic mechanisms $(27,28)$, which could be advantageous when tumor cells have accumulated mutations in apoptosis pathways. Moreover, through release of cytokines and chemokines as well as via crosstalk with, for instance, DCs, neutrophils may regulate adaptive immune responses $(25,29-33)$, potentially leading to in situ generation of long-term antitumor immunity. 
It has been demonstrated that neutrophils can induce tumor cell killing in the presence of IgG1 antitumor antibodies in vitro and in vivo $(34,35)$, but there is limited evidence that they play an important role in current $\mathrm{mAb}$ therapies in patients. However, their tumoricidal ability may be greatly enhanced by targeting the IgA Fc receptor Fc $\alpha$ RI (CD89), as it was shown that neutrophils kill tumor cells more effectively in the presence of IgA antibodies (28, 36-40). Importantly, Fc $\alpha$ RI but not Fc $\gamma$ R crosslinking resulted in neutrophil recruitment through release of leukotriene B4 (LTB4), leading to accumulation of neutrophils in 3-D tumor colonies in vitro (37). IgA does not activate NK cells because $\mathrm{Fc} \alpha \mathrm{RI}$ is not expressed.

To enlist a broader range of effector cells, combinations of IgG and IgA antibodies were tested in tumor models to induce tumor killing by both Fc $\gamma \mathrm{R}$ - and Fc $\alpha$ RI-bearing immune cells $(41,42)$. However, IgA has a short half-life, which hampers in vivo activity. In this study, we investigated how neutrophils, NK cells, and macrophages can be recruited as effector cells using a single therapeutic IgG1 bispecific antibody (BsAb) format that combined the agonistic activity of $\mathrm{Fc} \alpha \mathrm{RI}$ targeting with IgG1 characteristics, i.e., long half-life and induction of ADCC and ADCP.

\section{Results}

Generation and characterization of TrisomAbs. To augment neutrophil recruitment as effector cells for tumor therapy, we generated bispecific molecules (referred to as TrisomAb), in which the arms target Fc $\alpha \mathrm{RI}$ and a tumor-associated antigen (Figure 1A). TrisomAbs were generated via controlled Fab-arm exchange (FAE) DuoBody technology (43). Parental IgG1 antibodies against the tumor antigens EGFR and gp75 were of the G1m(f) allotype and contained the point mutation F405L, whereas parental IgG1 $\alpha$-Fc $\alpha$ RI antibodies were of the G1m(a) allotype and contained the K409R mutation in their respective $\mathrm{CH} 3$ domains (Figure 1A). Modified parental IgG1 antibodies were mixed and subjected to controlled reducing conditions in vitro that separated the 2 parental antibodies into half-molecules, which subsequently allowed reassembly and reoxidation to form TrisomAb BsAbs (Figure 1A). To verify FAE, we performed a sandwich ELISA utilizing the allotypic differences between the TrisomAbs and their parental forms. When an antibody against G1m(f) was used for both coating and detection, only the parental $\alpha$-EGFR mAb (cetuximab) containing the point mutation F405L [G1m(f) allotype] was detected (Figure 1B). Conversely, when antibodies against the G1m(a) allotype were used for both coating and detection, only the parental $\alpha$-Fc $\alpha$ RI $\mathrm{mAb}$ containing the K409R mutation was detected (Figure 1C). When $\alpha-G 1 m(f) m A b$ was used for coating and $\alpha-G 1 m(a) m A b$ as detection antibody, only TrisomAb $\alpha$-EGFR but neither parental form was detected, verifying the successful FAE of the parental antibodies (Figure 1D). Successful FAE of TrisomAb $\alpha$-gp75 was shown in a similar manner (Supplemental Figure 1, A-C).

To validate the binding specificity of TrisomAb $\alpha$-EGFR, we measured the binding of TrisomAb $\alpha$-EGFR to an Fc $\alpha$ RI, Fc $\gamma$ RIIIa (CD16a), as the most important Fc $\gamma$ R on NK cells and macrophages, and various EGFR-expressing tumor cell lines using a cellular surface plasmon resonance imaging (SPRi) platform. FcaRI was spotted on a streptavidin-coated sensor at different densities. Different antibodies, ligands, and cells were sequentially injected and subsequent binding was detected as response units (Figure 2A). A first flow with TrisomAb $\alpha$-EGFR showed binding to spotted Fc $\alpha$ RI, whereas IgG $\alpha$-EGFR in flow 1 did not (Figure 2, A and B). A second flow with soluble Fc $\gamma$ RIIIa showed binding to TrisomAb $\alpha$-EGFR (that had bound to FcaRI in flow 1; Figure 2, A and C). A second flow with soluble FcaRI showed no additional binding, indicating that spotted FcaRI was already occupied by binding of TrisomAb (Figure 2, A and C). Antibody binding affinity of TrisomAb $\alpha$-EGFR, human IgG1 $\alpha$-EGFR, and human IgG1 $\alpha$-Fc $\alpha$ RI for Fc $\alpha$ RI and human FcyR IIIa 158V protein is shown in Supplemental Table 1. Carcinoma cells with high (A431 cells) or intermediate (HTCT116 cells) EGFR expression but not SW621 cells (no EGFR expression) were able to bind to Fc $\alpha$ RI/Fc $\gamma$ RIIIa-occupied TrisomAb $\alpha$-EGFR during the third flow (Figure 2, A and D). Thus, TrisomAb $\alpha$-EGFR was able to simultaneously interact with FcaRI-, Fc $\gamma$ RIIIa-, and EGFR-positive tumor cells. To corroborate the binding specificity of TrisomAb $\alpha$-EGFR and TrisomAb $\alpha$-gp75 to neutrophils and cancer cells, we investigated the binding with flow cytometry. Trisom$\mathrm{Ab} \alpha$-EGFR bound to A431 cells and Fc $\alpha$ RI-positive neutrophils in a dose-dependent manner (Supplemental Figure 1D; supplemental material available online with this article; https://doi.org/10.1172/ JCI134680DS1). Both TrisomAb $\alpha$-EGFR and IgG $\alpha$-EGFR bound to A431 cells, whereas TrisomAb $\alpha$-gp75 and IgG $\alpha$-Fc $\alpha$ RI did not bind (Figure 2E). In addition, TrisomAb $\alpha$-EGFR, TrisomAb $\alpha$-gp75, and parental IgG $\alpha$-Fc $\alpha$ RI were able to bind to Fc $\alpha$ RIpositive neutrophils, whereas IgG $\alpha$-EGFR did not bind (Figure $2 \mathrm{~F}$ ). Similarly, only TrisomAb $\alpha$-gp75 bound B16F10gp75 cells (Figure $2 G)$. In conclusion, both TrisomAb $\alpha$-EGFR and TrisomAb $\alpha$-gp75 were able to bind to Fc $\alpha$ RI-positive neutrophils, Fc $\gamma$ RIIIa, and their specific tumor-associated antigen.

TrisomAb induces FcyR-mediated effector functions. To investigate whether TrisomAbs induce effective NK cell-mediated killing of cancer cells, we performed an ADCC assay with A431 or B16F10gp75 cells in the presence of TrisomAb, IgA, and IgG antibodies against EGFR or gp75. Within 4 hours, NK cells reduced the number of A431 cells up to $60 \%$ in the presence of $1 \mu \mathrm{g} / \mathrm{mL}$ TrisomAb $\alpha$-EGFR or IgG $\alpha$-EGFR (Figure $3 \mathrm{~A}$ ). At a lower concentration $(0.1 \mu \mathrm{g} / \mathrm{mL}), \operatorname{IgG} \alpha$-EGFR was more efficient than TrisomAb $\alpha$-EGFR (Figure 3A). IgA $\alpha$-EGFR was not able to induce ADCC (Figure 3A). Incubation of TrisomAb $\alpha$-EGFR and IgG $\alpha$-EGFR antibodies for 24 hours resulted in complete elimination of A431 cells by NK cells, whereas IgA $\alpha$-EGFR had no effect (Supplemental Figure 1E). In the presence of $1 \mu \mathrm{g} / \mathrm{mL}$, TrisomAb $\alpha$-gp75 or IgG $\alpha$-gp75 elicited effective NK cell-mediated ADCC, resulting in B16F10gp75 killing of approximately $60 \%$ after 4 hours (Figure 3B). Increasing the antibody dose to $10 \mu \mathrm{g} / \mathrm{mL}$ did not further increase the killing of B16F10gp75 cells, whereas at a lower dose $(0.1 \mu \mathrm{g} / \mathrm{mL}), \operatorname{IgG} \alpha-\mathrm{gp} 75$ was somewhat more efficient than TrisomAb $\alpha$-gp75 (Figure 3B). IgA $\alpha$-gp75 was ineffective at all concentrations. To investigate the kinetics of NK cell-mediated ADCC, we performed live-cell imaging using Lifeact mScarlettransduced A431 cells and fluorescently labeled NK cells. Because NK cells generally kill through induction of apoptosis, a fluorescent caspase-3/7 indicator was used. TrisomAb $\alpha$-EGFR and IgG $\alpha$-EGFR induced caspase-3/7 activation within 2 hours (Supplemental Videos 1 and 2 and Figure 3C). No apoptosis was induced in the absence of antibodies or in the presence of $\operatorname{IgA} \alpha$-EGFR 
A Human IgG1 bi-specific

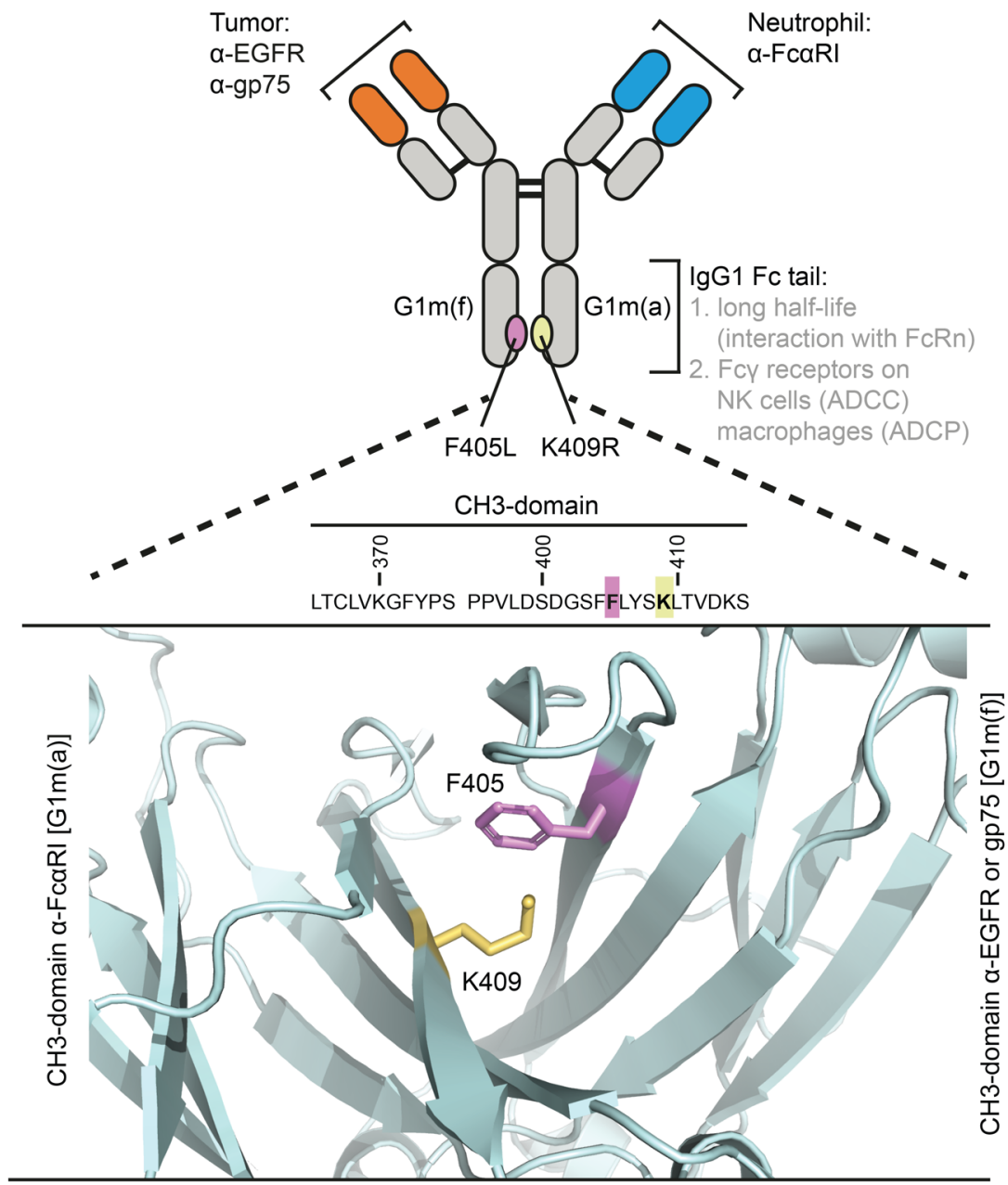

B

$$
\begin{aligned}
& - \text { hlgG1 } \alpha \text {-EGFR [G1m(f)] } \\
& - \text { - TrisomAb EGFR [G1m(a/f] } \\
& - \text { - hlgG1 } \alpha-F c a R I ~[G 1 m(a)]
\end{aligned}
$$

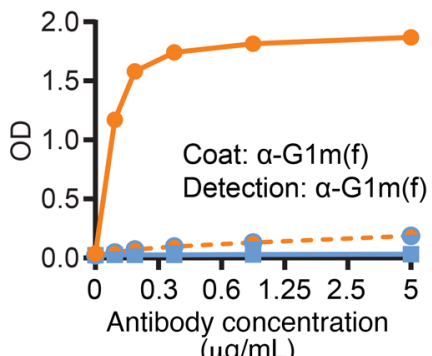

C

$$
\begin{aligned}
& - \text { hlgG1 } \alpha \text {-EGFR [G1m(f)] } \\
& -\odot \text { - TrisomAb EGFR [G1m(a/f] } \\
& - \text { hlgG1 } \alpha-F c a R I ~[G 1 m(a)]
\end{aligned}
$$

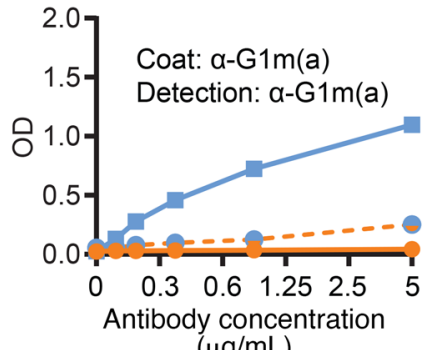

D

$$
\begin{aligned}
& \text { - - TrisomAb EGFR [G1m(a/f] } \\
& -- \text { hlgG1 a-FcaRI [G1m(a)] }
\end{aligned}
$$$$
\text { hlgG1 a-EGFR [G1m(f)] }
$$

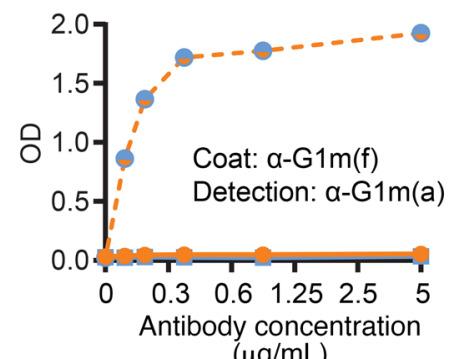

Figure 1. Generation of TrisomAb $\alpha$-EGFR and TrisomAb $\alpha$-gp75 by Fab-arm exchange. (A) Illustration of TrisomAb $\alpha$-EGFR and TrisomAb $\alpha$-gp75. TrisomAb is a bispecific human IgG1 antibody format targeting tumor-associated antigens with one arm and FcaRI with the other arm. Lower panel shows in silico model of the CH3-domain $\alpha$-FcaRI G1m(a)-CH3-domain $\alpha$-EGFR G1m(f) heterodimer interface. Residues F405 and K409 are highlighted in purple and gold, respectively. Image was generated from PBD ID 3AVE (79). Since the backbone is an IgG1 molecule, it has a long half-life and is capable of inducing Fc $\gamma \mathrm{R}$-mediated effector functions in NK cells and macrophages. (B-D) Sandwich ELISA showing successful Fab-arm exchange utilizing the allotypic differences between TrisomAb and its parental forms. Data show mean \pm SD of 1 representative example of more than 7 independent experiments B-D. 


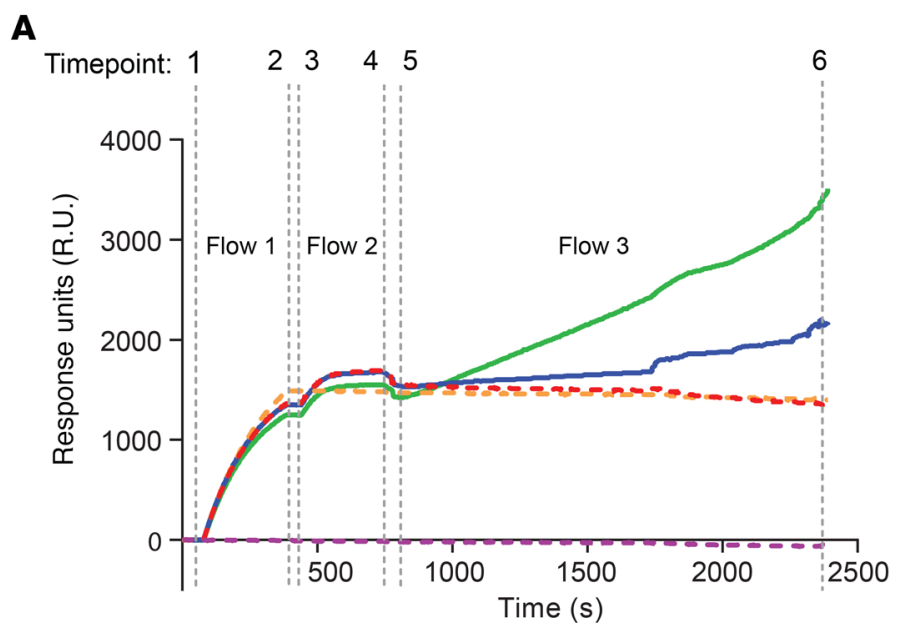

\begin{tabular}{|c|c|c|}
\hline Flow 1 & Flow 2 & Flow 3 \\
\hline TrisomAb a-EGFR & FcyRIIla & A431 \\
\hline TrisomAb a-EGFR & FcyRIIla & HCT116 \\
\hline TrisomAb a-EGFR & FcyRIIla & SW620 \\
\hline TrisomAb a-EGFR & FcaRI & Buffer \\
\hline IgG a-EGFR & FcaRI & Buffer \\
\hline
\end{tabular}

B

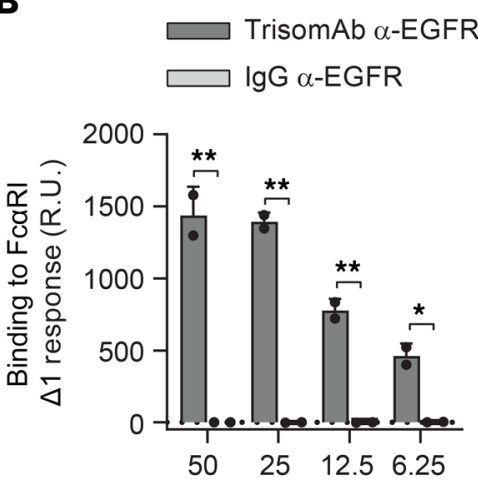

FcaRl spot density $(\mathrm{nm})$

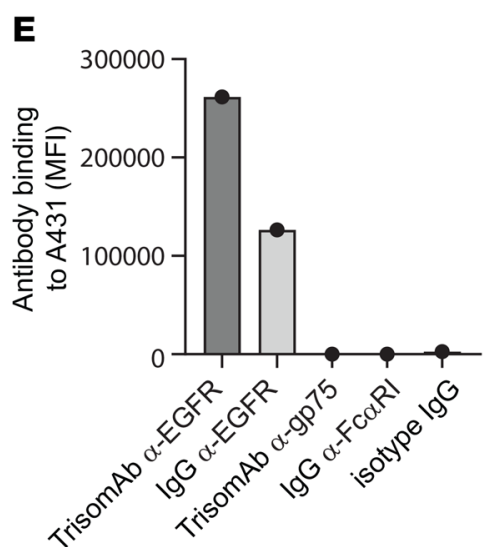

C

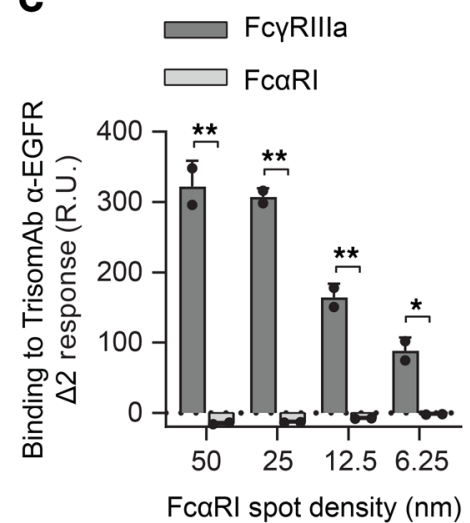

$\mathbf{F}$

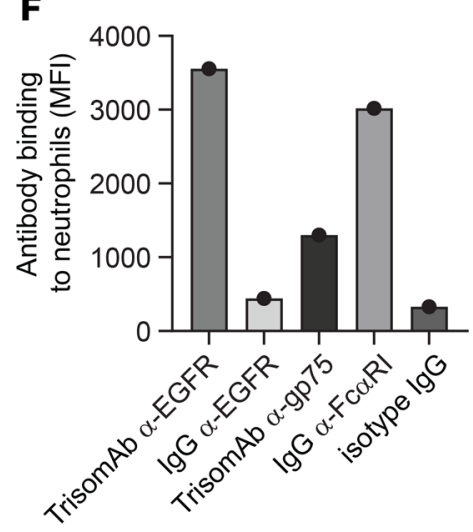

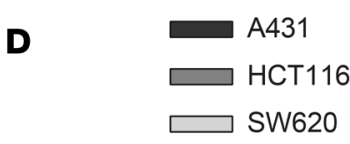

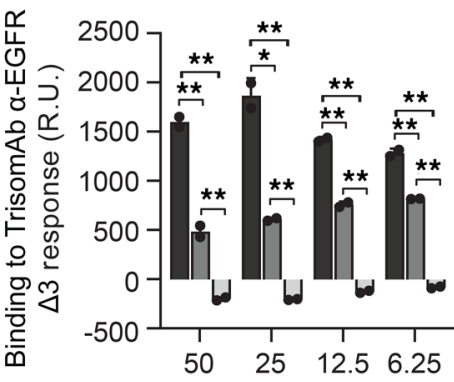

FcaRl spot density $(\mathrm{nm})$

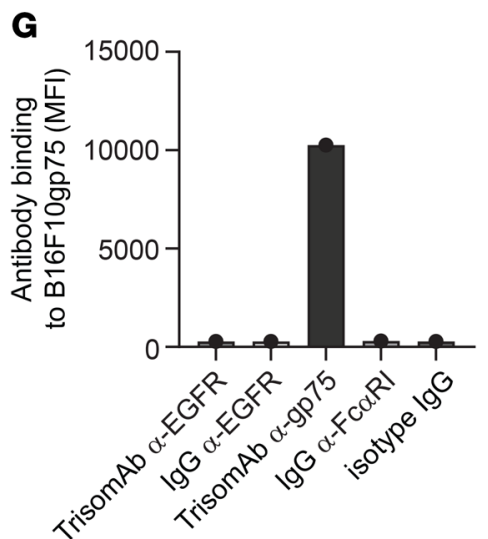

Figure 2. Binding specificity of EGFR, gp75, Fc $\alpha$ RI, and Fc $\gamma$ RIIla for TrisomAb $\alpha$-EGFR and TrisomAb $\alpha$-gp75. (A) Representative cellular surface plasmon resonance sensorgrams showing TrisomAb $\alpha$-EGFR, injected at a flow rate of $20 \mu \mathrm{L} / \mathrm{min}$, interacting with immobilized FcaRI (flow 1), Fc $\gamma R$ IIlla interacting with TrisomAb $\alpha$-EGFR bound to FcaRI (flow 2), and affinity of A431 (high EGFR expression), HCT116 (moderate EGFR expression), and SW620 cells (no EGFR expression) for TrisomAb $\alpha$-EGFR bound to FcaRI and Fc $\gamma$ RIIla (flow 3). Table on the right shows the injection summary of the sensorgram. (B) Quantification of $\Delta 1$ response (time point 2 - time point 1, flow 1). (C) Quantification of $\Delta 2$ response (time point 4 - time point 3, flow 2). (D) Quantification of $\Delta 3$ response (time point 6 - time point 5 , flow 3$)$. Binding of therapeutic $\mathrm{mAbs}(10 \mu \mathrm{g} / \mathrm{mL})$ to $\mathrm{A} 431$ cells $(\mathbf{E})$, neutrophils $(\mathbf{F})$, and B16F10gp75 cells $(\mathbf{C})$ measured by flow cytometry. Data in $\mathbf{A}-\mathbf{D}$ are from 2 independent experiments; error bars show SD. ${ }^{*} P<0.05$; ${ }^{*} P<0.01$ by multiple $t$ tests in $\mathbf{B}-\mathbf{D}$.

(Supplemental Videos 1 and 2 and Figure 3C). No differences in kinetics were observed between TrisomAb $\alpha$-EGFR or IgG $\alpha$-EGFR (Figure 3, C-E). Next, we investigated the efficacy of TrisomAb-dependent phagocytosis of A431 and B16F10gp75 cells by monocyte-derived macrophages. TrisomAb, IgA, and IgG $\alpha$-EGFR antibodies mediated ADCP of A431 cells in a dose-dependent manner, although IgA was slightly less efficient (Figure 3F). When B16F10gp75 cells were used as target cells, TrisomAb $\alpha$-gp75 performed better than IgA $\alpha$-gp75 and IgG $\alpha$-gp75 (Figure 3G). In conclusion, TrisomAb mediated effective ADCC and ADCP, demonstrating that the IgG heavy chain of the BsAb retained the ability to activate NK cells and macrophages through Fc $\gamma$ R. 
A

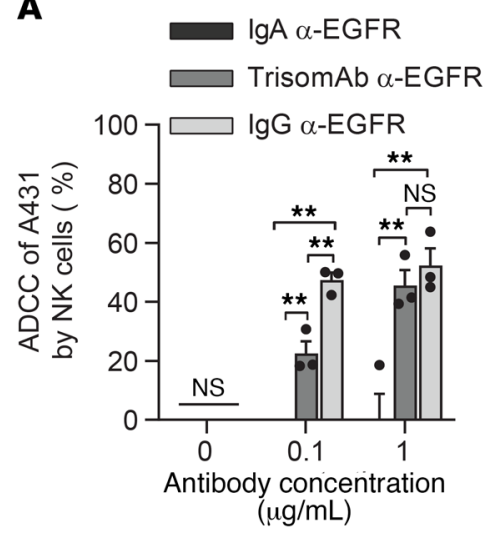

B

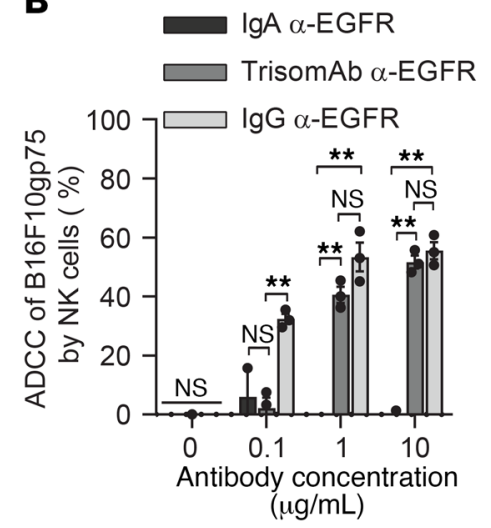

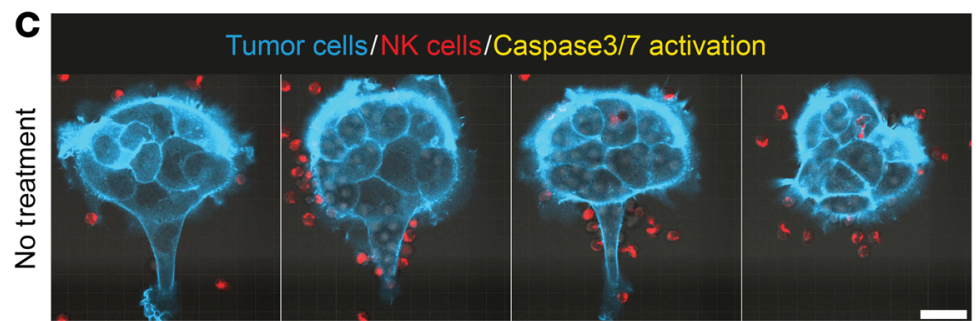
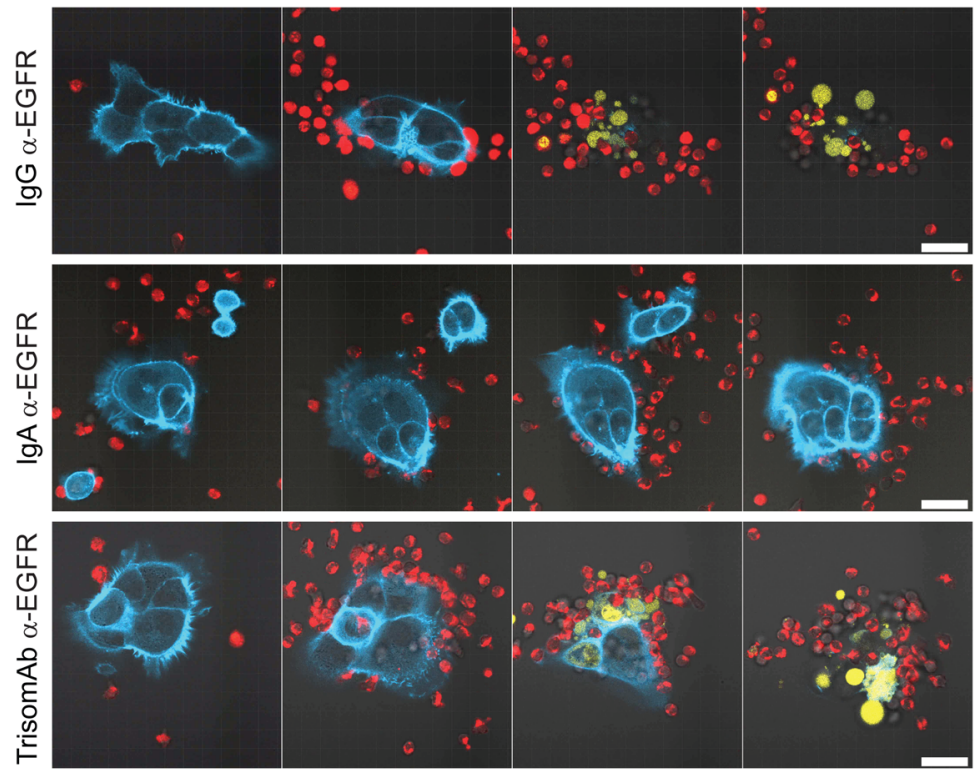

0
$1 \mathrm{~h}$
$2 \mathrm{~h}$

$\mathbf{F}$

IgA $\alpha$-EGFR

$\square$ TrisomAb $\alpha$-EGFR

$\square \operatorname{IgG} \alpha-E G F R$

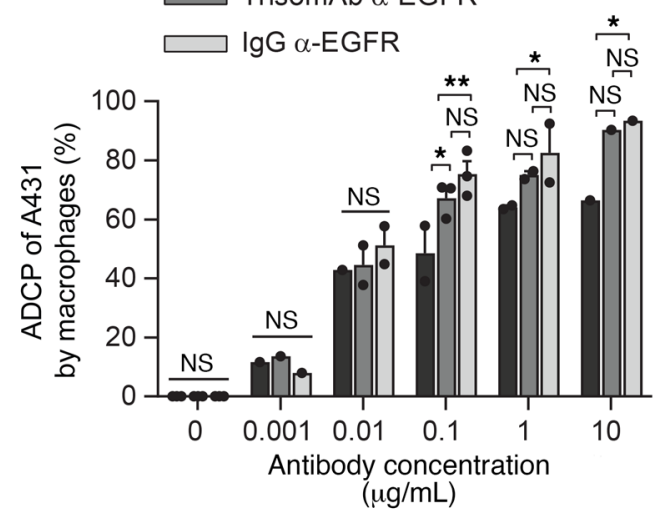

$(\mu \mathrm{g} / \mathrm{mL})$

D $\rightarrow$ No Treatment $\rightarrow$ TrisomAb $\alpha$-EGFR

$\simeq \operatorname{IgA} \alpha-$ EGFR $\quad \Rightarrow \lg G \alpha$-EGFR

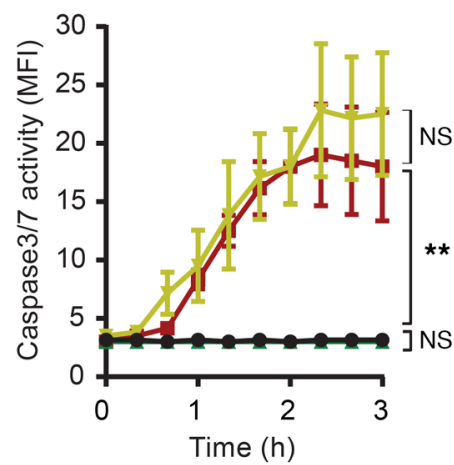

E

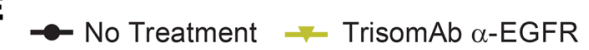

$\simeq \lg \alpha$-EGFR $\rightarrow \lg G \alpha$-EGFR

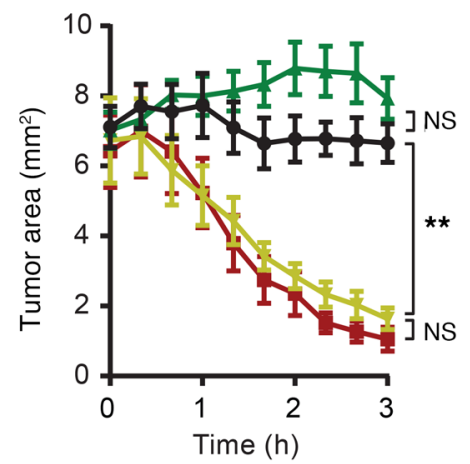


Figure 3. TrisomAb induces effective Fc $\gamma \mathrm{R}$ - and Fc $\alpha$ R-mediated ADCC and ADCP of cancer cells. (A) ADCC of A431 cells by NK cells (effector/ target 5:1) in the presence of $0,0.1$, and $1 \mu \mathrm{g} / \mathrm{mL}$ TrisomAb, IgA, and IgC $\alpha$-EGFR antibodies. CellTiter-Blue Cell Viability Assay was performed after 4 hours. (B) ADCC of B16F10gp75 cells by NK cells (E/T 5:1) in the presence of $0,0.1,1$, and $10 \mu \mathrm{g} / \mathrm{mL}$ TrisomAb, IgA, and IgG $\alpha$-gp75 antibodies. CellTiter-Blue Cell Viability Assay was performed after 4 hours. (C) Confocal live-cell imaging of antibody-mediated induction of apoptosis (caspase-3/7 activity, yellow) in A431 cells (blue) by NK cells (red). Scale bars: $25 \mu \mathrm{m}$. (D) Quantification of caspase-3/7 activity and (E) tumor area for different therapeutic antibodies. (F) ADCP of A431 cells by monocyte-derived macrophages in the presence of $0,0.001,0.01,0.1,1$, and $10 \mu \mathrm{g} / \mathrm{mL}$ TrisomAb, IgA, and IgG $\alpha$-EGFR antibodies. Percentage of phagocytic events was measured by flow cytometry 4 hours after incubation with macrophages at an effector to target ratio of 5:1. (C) ADCP of B16F10gp75 cells by monocyte-derived macrophages in the presence of 0, 0.01, 0.1, 1 and $10 \mu \mathrm{g} / \mathrm{mL}$ TrisomAb, IgA, and IgG $\alpha$-gp75 antibodies. Percentage of phagocytic events was measured by flow cytometry 4 hours after incubation with macrophages at an effector to target ratio of 5:1. Data are from 3 independent experiments; caspase-3/7 activation and tumor area were quantified for 8 tumor colonies (5-30 cells) per group; error bars show SEM. ${ }^{*} P<0.05 ;{ }^{*} P<0.01$ by 2 -way ANOVA with Tukey's multiple-comparison correction in $\mathbf{A}, \mathbf{B}$, and $\mathbf{D}-\mathbf{G}$.

TrisomAb induces neutrophil-mediated cytotoxicity through Fc $\alpha R I$. To determine whether TrisomAb is able to activate Fc $\alpha$ RI, we next investigated signaling after crosslinking of Fc $\alpha$ RI with TrisomAb and measured the amount of tyrosine phosphorylation, which is an early step in the signaling cascade. To exclude the potential contribution of the IgG Fc tail and Fc $\gamma \mathrm{R}$ signaling, RBL cells were used that had been transfected with human Fc $\alpha$ RI but did not express Fc $\gamma R$ (44). Clustering of Fc $\alpha$ RI with either the parental $\alpha$-Fc $\alpha$ RI mAb or TrisomAb $\alpha$-EGFR induced transient downstream signaling in RBL-FcaRI cells (Supplemental Figure 2, A-C and F). Elevated tyrosine phosphorylation was detected between 1 and 10 minutes, with strong activation of proteins sized approximately $40 \mathrm{kDa}$ and $70 \mathrm{kDa}$ (Supplemental Figure 2F). Incubation with IgG $\alpha$-EGFR (as control) or the secondary antibody alone did not induce tyrosine phosphorylation (Supplemental Figure 2, D-F). Similarly, crosslinking of Fc $\alpha$ RI on PMNs showed comparable tyrosine phosphorylation patterns to that observed in RBL-Fc $\alpha$ RI (Supplemental Figure 3, A-E). To exclude interference of signaling via FcaRI on monocytes, tyrosine phosphorylation after Fc $\alpha$ RI crosslinking was repeated in enriched neutrophil fractions (Supplemental Figure 4, A-E). Neutrophil enrichment was confirmed using cytospins and imaging (Supplemental Figure 5, A and B), and was increased from approximately $90 \%$ to $98 \%$ after the enrichment step (Supplemental Figure 5C). Thus, clustering of Fc $\alpha$ RI with TrisomAb $\alpha$-EGFR induced transient tyrosine signaling in RBL cells and neutrophils, demonstrating that TrisomAb $\alpha$-EGFR was able to induce Fc $\alpha$ RI-mediated signaling.

To investigate whether TrisomAb $\alpha$-EGFR was able to induce neutrophil-mediated cytotoxicity, A431 cells were incubated with PMNs in the presence of TrisomAb, IgA, or IgG $\alpha$-EGFR antibodies. Within a time period of 4 hours, PMNs reduced the number of tumor cells up to $90 \%$ in the presence of $10 \mu \mathrm{g} / \mathrm{mL}$ TrisomAb $\alpha$-EGFR, outperforming the parental IgG $\alpha$-EGFR mAb and similar to IgA $\alpha$-EGFR (Figure 4A). At lower doses, TrisomAb $\alpha$-EGFR was more efficient at inducing tumor cell killing than $\operatorname{IgG} \alpha$-EGFR, but slightly less effective compared with IgA $\alpha$-EGFR (Figure 4A). To exclude the possible contribution of contaminating monocytes, eosinophils, basophils, or mast cells in the PMN fraction, we examined the killing capacity of enriched neutrophils. No major differences were observed between elimination of tumor cells by PMNs or enriched neutrophils in the presence of IgG $\alpha$-EGFR, IgA $\alpha$-EGFR, or TrisomAb $\alpha$-EGFR (Supplemental Figure 5, D and E). Additionally, the presence of eosinophil- and basophil-related cytokines, i.e., IL-4 and IL-13, was not detected in the supernatants of ADCC assays (data not shown), nor was an increase in histamine levels observed (Supplemental Figure 5F), excluding a major confounding effect of eosinophils, basophils, and mast cells. Lactoferrin and IL-8 release in supernatants were evaluated as a measure for neutrophil degranulation and cytokine release, respectively. Lactoferrin release showed a similar pattern as neutrophil-mediated killing of A431 cells in the presence of the different antibodies (Figure 4B). IL-8 was detected in supernatants of conditions in which IgA $\alpha$-EGFR or TrisomAb $\alpha$-EGFR had been added, but not in IgG $\alpha$-EGFR-derived supernatants (Figure 4C). The presence of chemotactic stimuli in supernatants was further investigated with a chemotaxis assay. Some neutrophil recruitment was observed toward supernatants when IgG $\alpha$-EGFR antibodies were added (Supplemental Figure 5G). Chemotactic activity of supernatants was, however, greatly increased when either $\operatorname{IgA} \alpha$-EGFR or TrisomAb $\alpha$-EGFR were added in the killing assays (Supplemental Figure 5G). Killing assays with B16F10gp75 cells in the presence of TrisomAb $\alpha$-gp75 corroborated the data obtained with TrisomAb $\alpha$-EGFR. Addition of $10 \mu \mathrm{g} / \mathrm{mL}$ TrisomAb $\alpha$-gp75 or IgA $\alpha$-gp75 outperformed killing of tumor cells by PMNs in the presence of the parental IgG $\alpha$-gp75 mAb (Figure 4D). Lactoferrin release correlated with tumor cell death, and IL-8 was released in both supernatants of IgA $\alpha$-gp75 and TrisomAb $\alpha$-gp 75 conditions, although levels of IL-8 were lower in the latter condition (Figure 4, E and F). Together, these data indicate that TrisomAb induced enhanced killing of tumor cells, as well as increased neutrophil degranulation and release of chemotactic stimuli compared with the parental IgG antibodies.

TrisomAb induces neutrophil swarming. To study dynamic contacts between neutrophils and tumor cells in real time, we performed live-cell imaging followed by in-depth cellular tracking analyses. Real-time recordings of neutrophil-mediated killing showed neutrophil swarming toward A431 cells in the presence of IgA $\alpha$-EGFR and TrisomAb $\alpha$-EGFR (Supplemental Videos 3 and 4 , and Figure 5A). The number of neutrophils interacting with tumor cells was quantified with a custom written Xtension for Imaris (Supplemental Video 5). TrisomAb $\alpha$-EGFR triggered swarming behavior more rapidly than IgA $\alpha$-EGFR (Figure 5, A-C). Nonetheless, killing of A431 cells after 40 minutes was comparable, as indicated by the reduction in tumor area (Figure 5, A and D). During killing assays, we observed long stretches of F-actin in tumor cells as well as uptake of cancer cell-derived F-actin by neutrophils (Supplemental Video 6 and Figure 5E), which is a process referred to as trogocytosis (35). Quantification showed that TrisomAb $\alpha$-EGFR and IgA $\alpha$-EGFR induced trogocytosis 3 times more efficiently compared with IgG $\alpha$-EGFR (Figure 5F). Thus, TrisomAb induced neutrophil swarming and killing (trogocytosis) through FcaRI in a similar manner as IgA antitumor antibodies. 

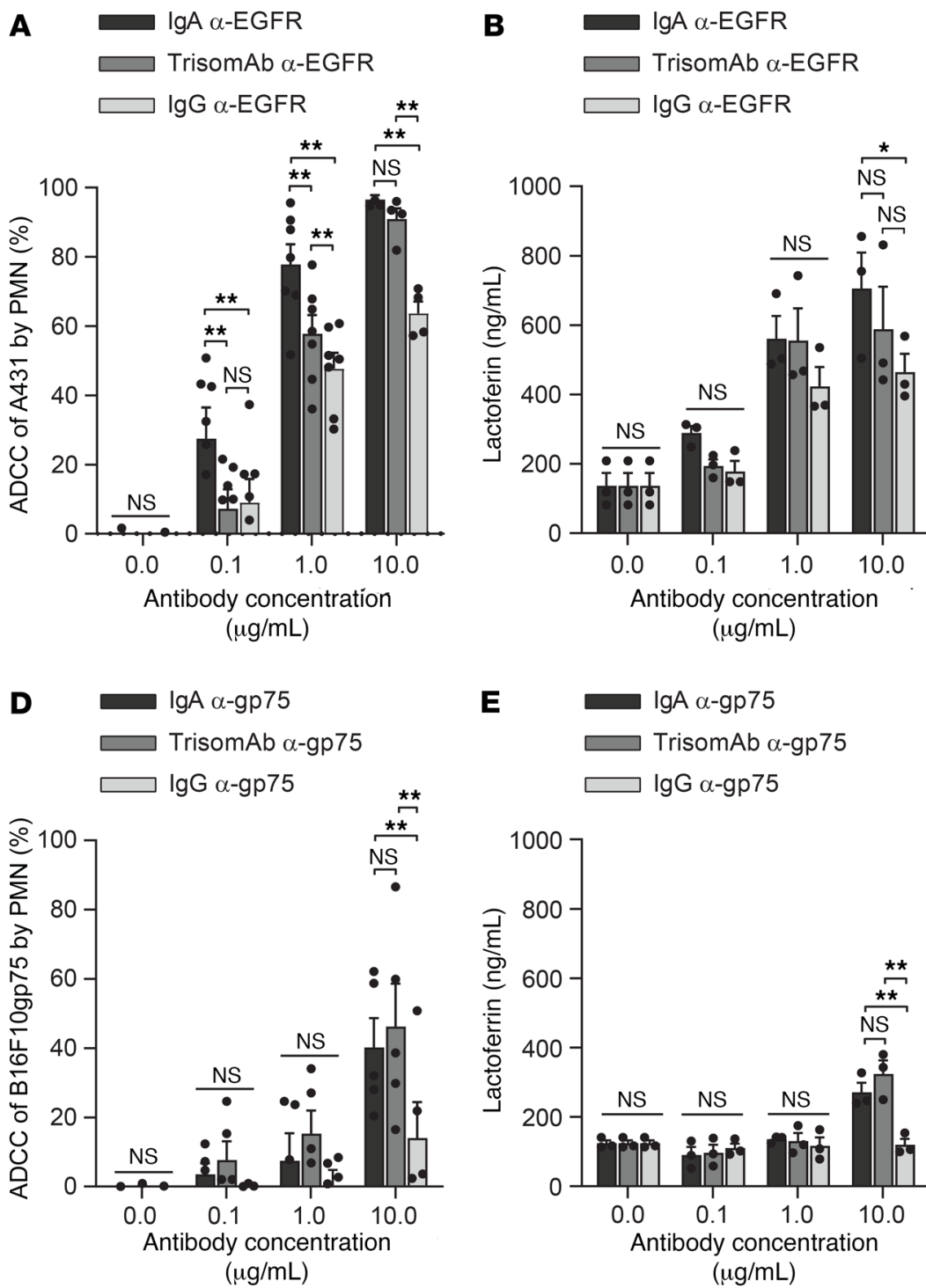

$\mathbf{E}$
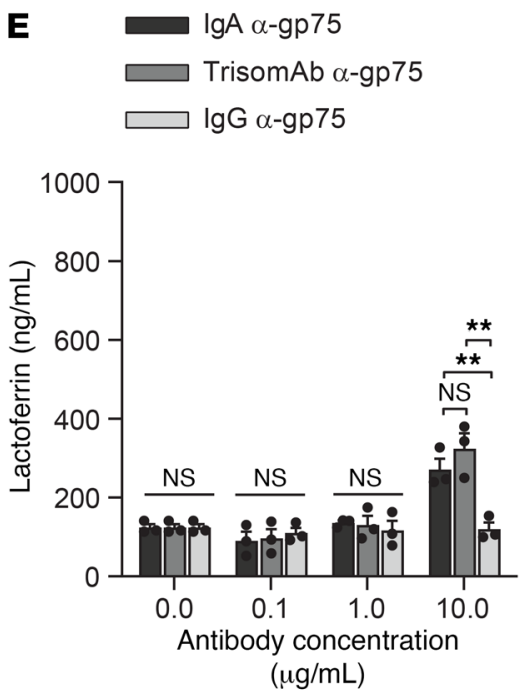

C
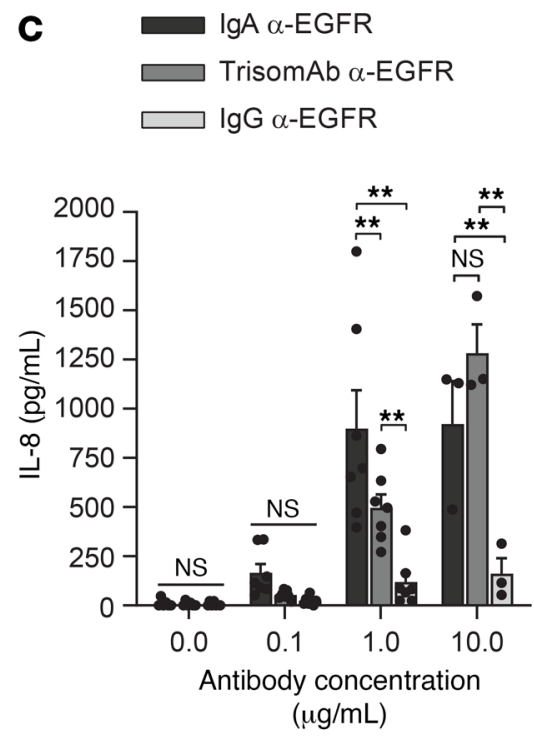

$\mathbf{F}$
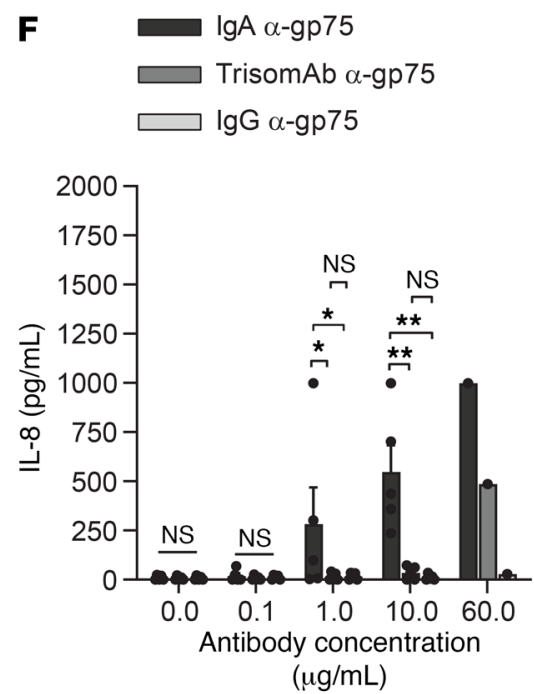

Figure 4. Trisom Ab induces lactoferrin and IL-8 secretion during PMN-mediated killing. (A) ADCC of A431 cells by neutrophils (effector/target 50:1) in the presence of $0,0.1,1$, and $10 \mu \mathrm{g} / \mathrm{mL}$ TrisomAb, IgA, and IgG $\alpha$-EGFR antibodies. CellTiter-Blue Cell Viability Assay was performed after 4 hours. (B) Lactoferrin detection in supernatant from ADCC assay shown in A measured by ELISA. (C) IL-8 detection in supernatant from ADCC assay shown in A measured by ELISA. (D) ADCC of B16F10gp75 cells by neutrophils (effector/target 50:1) in the presence of 0, 0.1, 1, and 10 $\mu$ g/mL TrisomAb, IgA, and IgC $\alpha$-gp75 antibodies. CellTiter-Blue Cell Viability Assay was performed after 4 hours. (E) Lactoferrin detection in supernatant from ADCC assay shown in $\mathbf{D}$ measured by ELISA. (F) IL-8 detection in supernatant from ADCC assay shown in $\mathbf{D}$ measured by ELISA. Data are compiled from 3-7 independent experiments in A-F; error bars show SEM. ${ }^{*} P<0.05 ;{ }^{*} P<0.01$ by 2 -way ANOVA with Tukey's multiple-comparison correction in $\mathbf{A}-\mathbf{F}$.

Because TrisomAb has the ability to recruit and activate NK cells, macrophages, and neutrophils, we next investigated killing of tumor cells by WBCs containing neutrophils, monocytes, and NK cells. The tumor killing capacity of WBCs was comparable to that of PMNs, probably because PMNs constitute the majority of WBCs. No additive or synergistic effect of PBMCs was measurable, most likely because of the relatively high neutrophil numbers in relation to NK cell numbers and limited access of tumor cells in the presence of an overwhelming number of WBCs (Supplemental Figure $6, \mathrm{~A}-\mathrm{C}$ ). To overcome these issues, cytotoxicity assays were also performed with isolated PMNs and NK cells. Simultaneous recruitment of NK cells and neutrophils enhanced tumor killing, which was similar to the sum of both cell types alone (Figure 6A).
Cancer patient-derived neutrophils mediate effective killing in the presence of TrisomAb $\alpha$-EGFR. One study has described the immunosuppressive phenotype of neutrophils in patients with cancer (45). To assess whether neutrophils of patients with colorectal cancer were able to kill tumor cells, killing assays were performed in the presence of TrisomAb, IgA, or IgG $\alpha$-EGFR antibodies. IgG $\alpha$-EGFR-mediated killing of A431 cells by patient neutrophils was poor compared with killing by neutrophils of healthy donors (Figure 6B; dashed line 2 represents the average killing of A 431 cells by neutrophils from healthy donors in the presence of $10 \mu \mathrm{g} / \mathrm{mL}$ IgG $\alpha$-EGFR). Importantly, killing of A431 cells by patient neutrophils in the presence of TrisomAb $\alpha$-EGFR and IgA $\alpha$-EGFR antibodies was comparable to killing by neutrophils of healthy individuals 
A
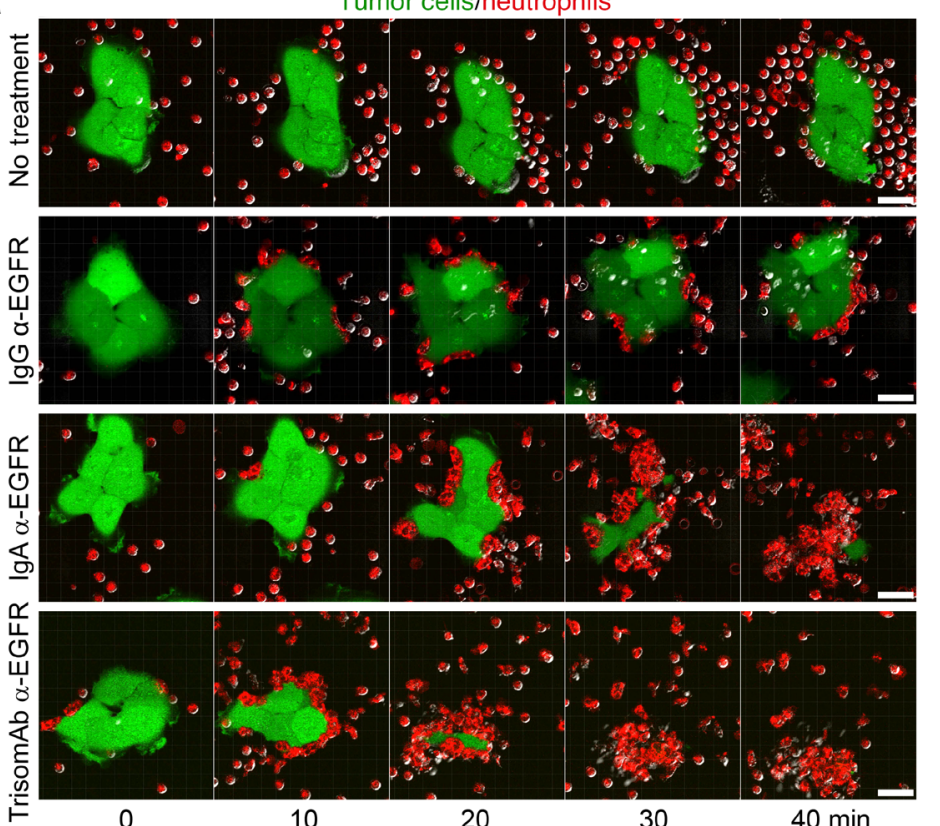

10

\section{C}

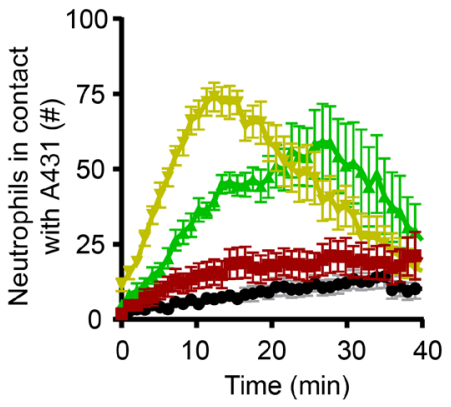

E

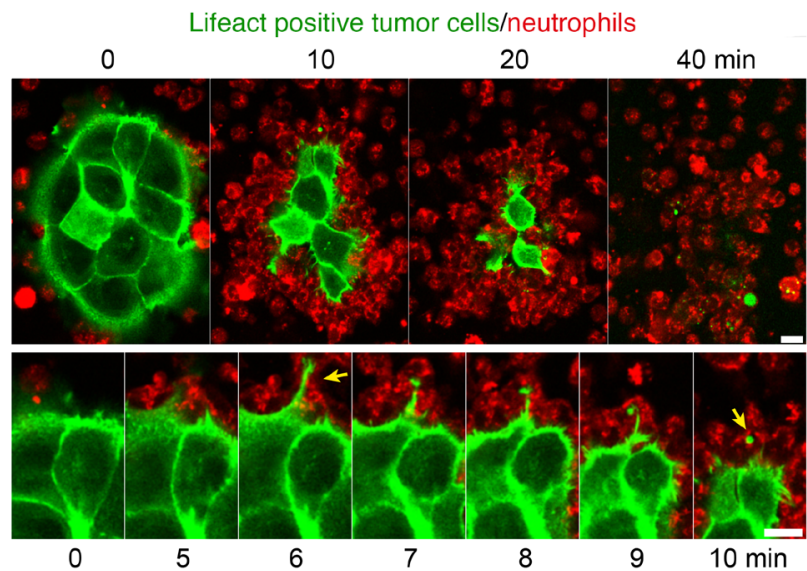

D

$\mathbf{F}$
B
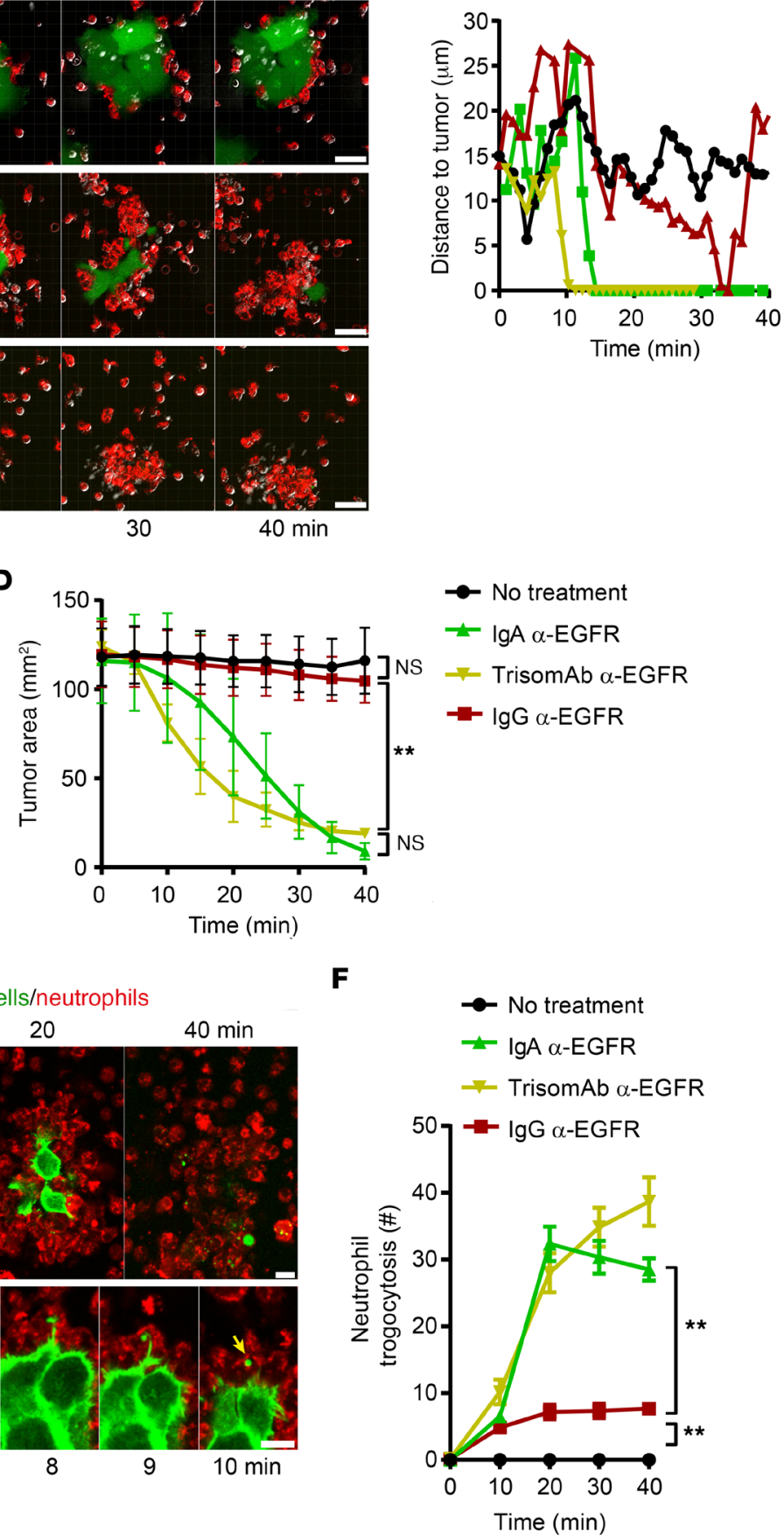

Figure 5. TrisomAb induces neutrophil swarming. (A) Time-lapse images of A431 colonies (green) and neutrophils (red) recorded with a Nikon Eclipse Ti confocal microscope showing neutrophil swarming toward A431 cells in the presence of $2 \mu \mathrm{g} / \mathrm{mL}$ TrisomAb, IgA, IgG $\alpha$-EGFR, or no antibody. Scale bars: 25 $\mu \mathrm{m}$. (B) Quantification of neutrophil distance to A431 cells (in $\mu \mathrm{m}$ ) over time. (C) Quantification of neutrophil contact with A431 cells over time. (D) Quantification of tumor area (in $\mathrm{mm}^{2}$ ) during neutrophil-mediated cytotoxicity. (E) Time-lapse images of neutrophil (red) trogocytosis during Lifeact-positive A431 cell (green) killing using various therapeutic mAbs. Upper panel shows overview image of neutrophil swarming, trogocytosis, and tumor cell death over time. Bottom panel shows zoom of a single trogocytosis event. Arrow at 6 minutes indicates the start and arrow at 10 minutes indicates the end of the event. Scale bars: $10 \mu \mathrm{m}$. (F) Quantification of neutrophil trogocytosis. Data are compiled from 4 independent experiments with more than 10 tumor colonies (5-20 cells) per group in A-D and 6 tumor colonies (5-20 cells) per group in $\mathbf{E}$ and $\mathbf{F}$; error bars show SEM. ${ }^{* *} P<0.01$ by 2 -way ANOVA with Tukey's multiple-comparison correction in $\mathbf{D}$ and $\mathbf{E}$. 
(Figure 6B; dashed line 1 represents the average killing of A431 cells by neutrophils from healthy donors in the presence of $10 \mu \mathrm{g} /$ mL IgA $\alpha$-EGFR and TrisomAb $\alpha$-EGFR). Lactoferrin release correlated with tumor cell death, and IL-8 was released in supernatants of IgA $\alpha$-EGFR and TrisomAb $\alpha$-EGFR conditions (Figure 6, $\mathrm{C}$ and $\mathrm{D})$. Together, these data indicate that neutrophils of cancer patients retained the capacity to kill tumor cells when targeted with TrisomAb or IgA $\alpha$-EGFR antibodies.

TrisomAb half-life and efficacy in vivo. Motivated by the promising in vitro results of TrisomAb, we explored the in vivo efficacy of TrisomAb in a syngeneic mouse model using B16F10gp75 melanoma in immunocompetent C57BL/6 mice. To investigate halflife, mice were injected with $50 \mu \mathrm{g}$ TrisomAb, IgG, or IgA $\alpha$-gp75 antibodies. High TrisomAb $\alpha$-gp75 and IgG $\alpha$-gp75 antibody titers were observed in the blood 6 hours after injection, which remained stable up to 8 days (Figure 7A). However, the majority of IgA $\alpha$-gp75 was already removed from the circulation after 6 hours, consistent with the poor half-life of human IgA in mice. The half-life of TrisomAb $\alpha$-gp75 and IgG $\alpha$-gp75 was approximately 8 days, whereas the half-life of IgA $\alpha$-gp75 was about 15 hours (Figure 7A). Mice developed mouse $\alpha$-human antibodies (MAHAs) after 11 days, resulting in concomitant clearance of TrisomAb $\alpha$-gp75 and $\operatorname{IgG} \alpha$-gp75 antibodies after day 13 (Supplemental Figure 7, A and D). Mice injected with PBS showed no MAHA development (data not shown).

We next assessed the efficacy of TrisomAb $\alpha$-gp75 therapy in vivo by measuring outgrowth of s.c.-injected B16F10gp75 cells in Fc $\alpha$ RI transgenic C57BL/6 mice (46). TrisomAb $\alpha$-gp75 treatment significantly reduced tumor outgrowth in Fc $\alpha$ RI transgenic mice compared with IgG $\alpha$-gp75 treatment, which had minimal therapeutic response (Figure 7B). No difference between TrisomAb $\alpha$-gp75 and IgG $\alpha$-gp75 treatment was observed in nontransgenic littermates. In addition, we examined survival over time. Mice treated with PBS or hIgG1 $\alpha$-gp75 antibodies failed to reject the B16F10gp75 tumors and did not survive beyond day 21 (Figure 7C). Tumors in the mice that had received TrisomAb developed more slowly, resulting in longer survival before the human endpoint was reached. Furthermore, approximately $20 \%$ of the mice achieved complete tumor rejection after TrisomAb treatment (Figure 7C). To identify which immune cell types contributed to the elimination of tumor cells in vivo, we performed tumor growth experiments after neutrophils, NK cells, or macrophages had been depleted with depleting antibodies. Depletion of either NK cells, macrophages, or neutrophils reduced the therapeutic activity of TrisomAb treatment, indicating that all 3 immune cell types, i.e., macrophages, NK cells, and neutrophils, contributed to TrisomAb-induced tumor killing (Figure 7, D-F, and Supplemental Figure 8).

Taken together, these data showed that TrisomAb combines the advantages of IgG and Fc $\alpha$ RI targeting, establishing a single human IgG1 BsAb format capable of eradicating tumor cells using NK cells, macrophages, and neutrophils as effector cells. Moreover, TrisomAb has a half-life comparable to that of IgG1 and is more effective in reducing tumor development in vivo.

\section{Discussion}

In clinical practice, IgG therapeutic antibodies can have direct effects on tumor growth, activate the classical complement path- way that inserts the lytic membrane attack complex in the tumor cell membrane, or initiate NK cell-mediated ADCC and macrophage-mediated ADCP. However, IgG does not efficiently recruit neutrophils as effector cells. The latter might be achieved by the use of IgA antibodies, which can mediate neutrophil migration and promote activation. Because NK cells and macrophages have no or very low expression of FcaRI, IgA does not activate NK cells and ADCP is less efficient. Moreover, IgA does not bind to the neonatal FcR (FcRn), resulting in faster serum clearance and shorter half-life compared with $\operatorname{IgG}(47,48)$. We have now demonstrated that replacing one of the tumor-recognizing variable domains of cetuximab or $\alpha$-gp75 mAbs with an Fc $\alpha$ RI recognition domain enhanced IgG1-based cancer therapeutics with neutrophilmediated cytotoxicity. Importantly, IgG1 intrinsic effector capacities (i.e., long-half life, NK cell-mediated ADCC and ADCP by macrophages) are maintained.

Immunotherapy of cancer is predominantly focused on $\mathrm{CD}^{+} \mathrm{T}$ cell responses to tumors (49). However, in vivo studies have also described key roles for innate immune effector cells in tumor therapy. Studies have found that NK cells enhance antibody tumor targeting through release of chemokines and IFN- $\gamma$, increasing myeloid activation and infiltration, thereby supporting tumor elimination $(50,51)$. Furthermore, Kupffer cells in the liver efficiently phagocytosed circulating tumor cells after mAb therapy (14). Moreover, it was shown that the activity of human $\alpha$-CTLA-4 antibodies depended on Fc-mediated effector functions by myeloid cells in a mouse model expressing human Fc $\gamma$ Rs (16). In addition, the antitumor efficacy of combination immunotherapy (i.e., a vaccine combined with IL-2, a tumor-targeting antibody, and checkpoint inhibition) required the presence of neutrophils, macrophages, and NK cells to establish long-term anticancer immunity (32).

Previous studies have described neutrophils as potential effector cells in antibody-based cancer therapy. Initial studies were focused on targeting the high-affinity receptor for IgG (Fc $\gamma$ RI CD64; refs. 52, 53). However, Fc $\gamma$ RI phase I/II clinical trials were disappointing and efforts with Fc $\gamma$ RI bispecific antibodies were abandoned $(54,55)$. Alternatively, FcaRI has been identified as a candidate target molecule for tumor therapy $(56,57)$. Several Fab-based BsAbs targeting FcaRI and a range of different tumorassociated antigens (i.e., EGFR, HLA class II, CD30, HER-2/ neu, and EpCAM) have been described (26, 28, 37, 38, 42, 57, 58). Although these FcaRI BsAbs effectively induced neutrophil recruitment into tumor colonies in vitro with subsequent destruction, half-life was short, hampering in vivo validation of therapeutic activity. In addition, in the presence of human IgA $\alpha$-EGFR antibodies, neutrophils induce potent short-term antitumor responses in vitro $(36,40)$ and in vivo (39). However, like FcaRI Fab-based BsAbs, IgA antibodies have a short half-life, which may limit clinical use (47). To improve the half-life of IgA, antibody engineering has resulted in various fusion antibodies where (parts of) the heavy chain of IgA2 was linked to IgG1 (5961). For instance, an IgGA $\alpha$-HER-2/neu "cross-type" antibody induced both neutrophil- and macrophage-mediated killing of tumor cells in vitro (60). Similarly, a tandem IgG/IgA2 $\alpha$-HER-2/ neu antibody had improved half-life and induced tumor cell killing in vitro (59). Furthermore, an $\alpha$-CD20 antibody that contained 
A
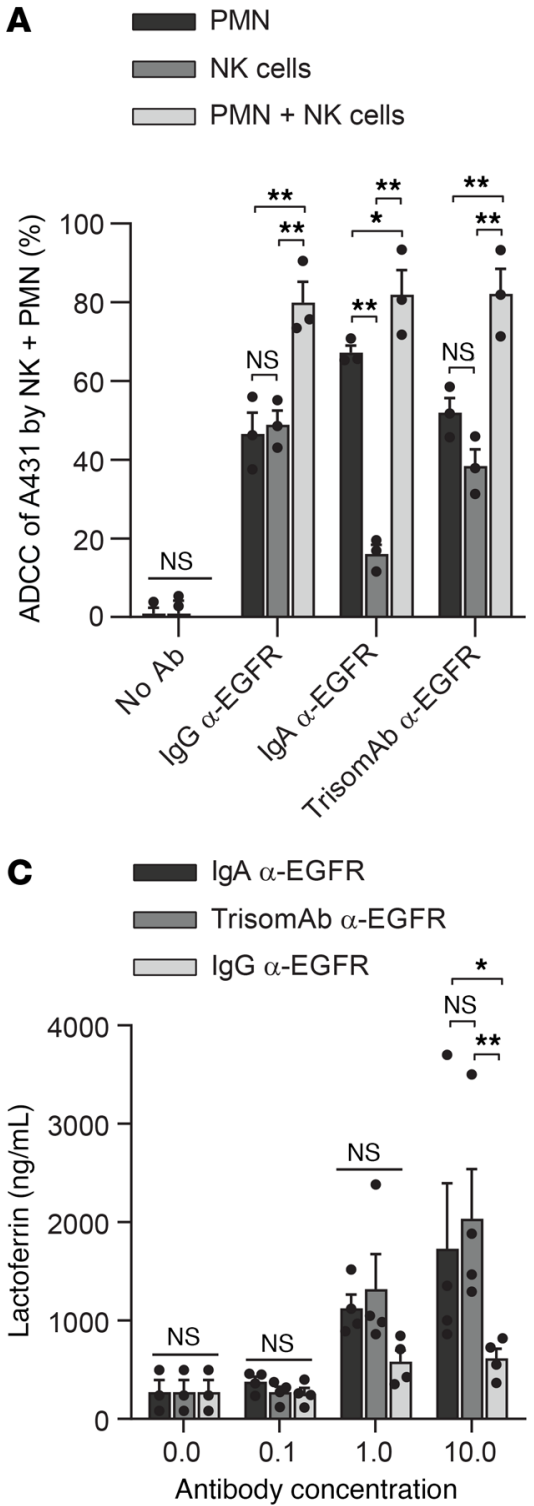

( $\mathrm{mg} \mathrm{mL}$ )
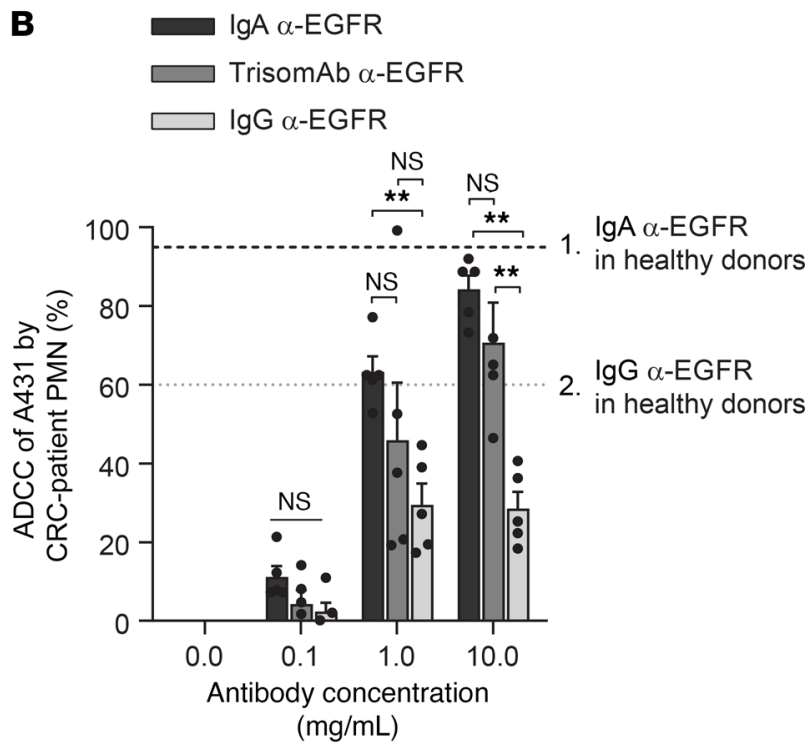

D
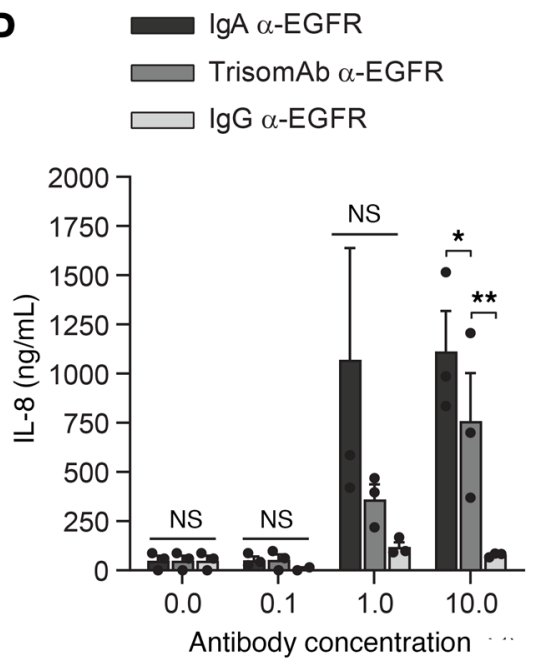

( $\mathrm{mg} \mathrm{mL}$ )

Figure 6. Added killing capacity of PMNs and NK cells in the presence of TrisomAb and cytotoxicity with colorectal cancer patient-derived neutrophils. (A) ADCC of A431 cells by NK cells (effector/target 5:1) and neutrophils (effector/target 50:1) in the presence of $1 \mu \mathrm{g} / \mathrm{mL}$ TrisomAb, IgA, and IgC $\alpha$-ECFR antibodies. CellTiter-Blue Cell Viability Assay was performed after 24 hours. (B) ADCC of A431 cells by colorectal cancer patient-derived neutrophils (effector/target 50:1) in the presence of increasing concentrations of TrisomAb, IgA, and IgG $\alpha$-EGFR antibodies. CellTiter-Blue Cell Viability Assay was performed after 4 hours. (C) Lactoferrin detection in supernatant from ADCC assay shown in B measured by ELISA. (D) IL-8 detection in supernatant from ADCC assay shown in B measured by ELISA. Data are compiled from $3(\mathbf{A})$ and 5 colorectal cancer patients (B), and 3-4 independent experiments (C and D). ${ }^{*} P<0.05 ;{ }^{*} P<0.01$ by 2-way ANOVA with Tukey's multiple-comparison correction in A-D.

both IgG and IgA Fc domains induced increased macrophagemediated antitumor activity in vivo (61). We demonstrated that TrisomAb has a comparable half-life to that of IgG1 but induces a significantly higher decrease in tumor outgrowth in vivo. Moreover, TrisomAb initiated NK cell-mediated ADCC and ADCP by macrophages. Additionally, tumor cell killing by neutrophils was induced along with neutrophil swarming.

We previously reported that neutrophils migrated toward SK-BR3 breast cancer cell colonies in a 3-D culture system in the presence of a Fab-fragment-based Fc $\alpha$ RI $\times$ HER2/neu (62). It was demonstrated that neutrophil swarming behavior induced by focal tissue damage depends on the release of chemotactic factors with a prominent role for LTB4 (63). FcaRI crosslinking results in the release of LTB4 (64). Additionally, neutrophils also release IL-8, which may further increase neutrophil recruitment from the circulation to the tumor, as it was shown that IL-8 increases endothelial adhesion molecules (37). Neutrophil swarming behavior resulted in close contact with tumor cells, referred to as the immunological or cytotoxic synapse (65). However, what killing mechanism(s) follow(s) synapse formation is a topic of ongoing debate. Several mechanisms have been described by which neutrophils kill antibody-opsonized tumor cells, which include degranulation, 
A
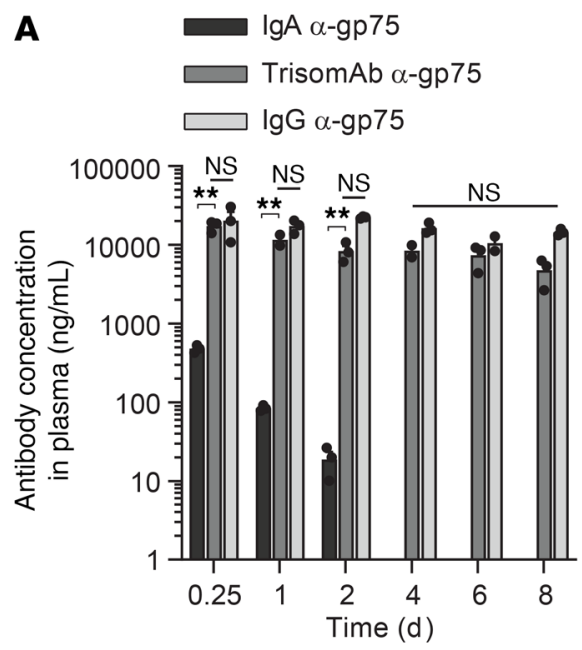

C

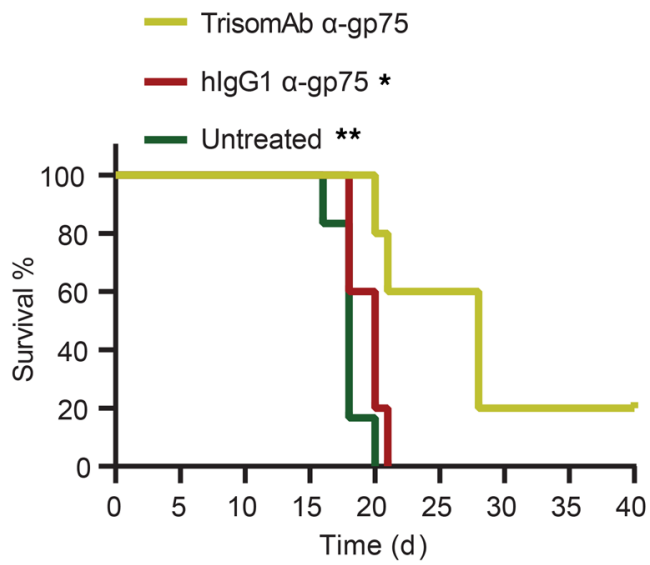

E

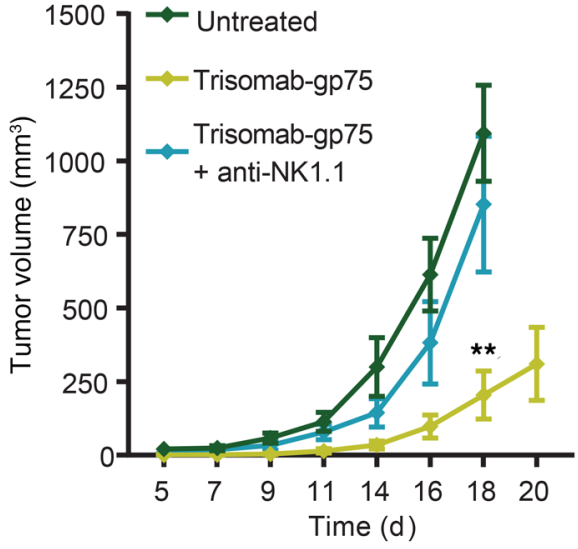

B
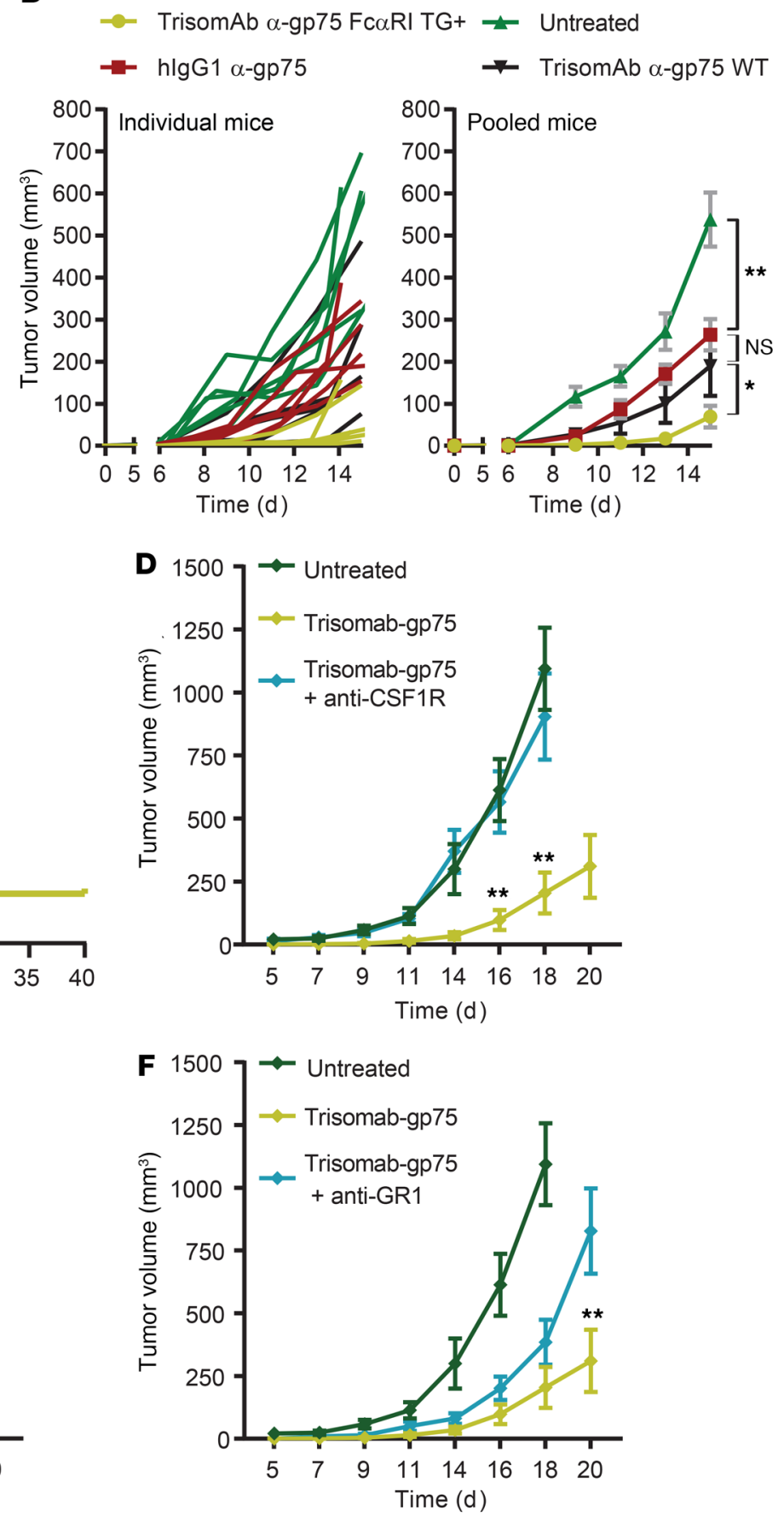

Figure 7. TrisomAb-induced tumor cell killing in vivo is dependent on macrophages, NK cells, and neutrophils. (A) Antibody concentration over time in serum of C57BL/6 mice i.p. injected with $50 \mu$ g therapeutic antibodies. (B) Tumor growth over time in mice s.c. inoculated with $1 \times 10^{4}$ B16F10gp 75 cells in the flank and treated with $100 \mu \mathrm{g}$ mAbs on days 0,1 , and 2. (C) Survival over time of mice s.c. inoculated with $1 \times 10^{4}$ B16F 10 gp 75 cells in the flank and treated with $100 \mu \mathrm{g}$ mAbs on days 0,1 , and 2. (D-F) Tumor growth over time with depleting antibodies specific for the indicated surface markers to deplete macrophages (CSF1R) (D), NK cells (NK1.1) (E), or neutrophils (Gr-1) (F). In A, $n=15$ C57BL/6 mice per group, $n=3$ for each time point. In B-F, 5-6 C57BL/6 mice per group; error bars showing SEM. ${ }^{*} P<0.05,{ }^{*} P<0.01$ versus TrisomAb by 2 -way ANOVA with Tukey's multiple-comparison correction in $\mathbf{A}$, B, and D-F; ${ }^{*} P<0.05 ;{ }^{* *} P<0.01$ versus TrisomAb by log-rank (Mantel-Cox) test in C.

release of neutrophil extracellular traps, apoptosis, autophagic cell death, necrosis, and phagocytosis $(27,28)$. Trogocytosis has been linked to tumor killing, representing a dominant mode of action during IgG1-dependent elimination of SK-BR3 breast cancer cells (35) and, more recently, for tumor cell killing in the presence of
IgA antibodies (66). Trogocytosis-induced cell death, a process referred to as trogoptosis, involves intimate contact between neutrophils and antibody-opsonized tumor cells, after which neutrophils mechanically disrupt the cancer cell plasma membrane, leading to a lytic nonapoptotic type of cancer cell death. In line with 
trogoptosis, we observed long stretches of F-actin in tumor cells, which were in contact with neutrophils, that only occurred under tension prior to internalization of cancer cell-derived F-actin by neutrophils. The quick leakage of calcein from tumor cells that was observed during live-cell imaging underscores the lytic cell death mechanism, which is in line with earlier observations that described the occurrence of necrotic cell death (24). Release of tumor antigens during necrotic types of cell death may boost the initiation of adaptive immune responses, as this may mediate cross-presentation by DCs $(29-32,67)$. Boosting neutrophil cytotoxicity by targeting Fc $\alpha$ RI with TrisomAb could therefore improve the induction of efficient adaptive anticancer responses because this results in abundant release of tumor antigens. Neutrophils furthermore release inflammatory chemokines that induce DC maturation, facilitating T cell responses $(31,33,34,68)$.

In summary, we have demonstrated that hIgG1-based cancer therapeutics can be extended with neutrophil-mediated cytotoxicity by replacing one of the tumor-recognizing variable domains with an Fc $\alpha$ RI recognition domain. In light of the additive value of neutrophil-mediated cytotoxicity and the supportive role of innate cells to facilitate long-term anticancer immunity, we anticipate that the TrisomAb format might improve antibody-based cancer immunotherapy, providing potential alternatives for cancer patients who do not benefit from current IgG mAb therapy.

\section{Methods}

Mice. Human FcaRI-transgenic mice (46) were crossed with LysMEGFP mice (69). Mice were bred and maintained at the Central Animal Facility of the VU University Medical Center (Amsterdam, the Netherlands). All mice had free access to regular chow and water. LysMEGFP mice were maintained as homozygous and Fc $\alpha$ RI as heterozygous strains. Fc $\alpha$ RI transgene-negative littermates of LysM C57BL/6 mice were used as controls. For all experiments, age-matched (predominantly 8-12 weeks old) male or female mice were used.

Cell cultures. Human colon carcinoma cell lines HCT116 (ATCC, CCL-247) and SW620 (ATCC, CCL-227) and RBL-hCD89 cells (gift of Renato C. Monteiro, Faculté de Médecine, Université Paris Diderot, Paris, France) (44) were cultured in DMEM (Gibco, 41966029) containing $10 \%(\mathrm{v} / \mathrm{v})$ heat-inactivated FCS (Biowest, S1810-500), $2 \mathrm{mM}$ L-glutamine (Gibco, A2916801), and $100 \mathrm{U} / \mathrm{mL}$ penicillinstreptomycin (Gibco, 15140122), hereafter referred to as complete DMEM. Human epidermoid carcinoma A431 cells were purchased from ATCC (CRL-2592) and cultured in complete DMEM. Murine melanoma B16F10gp75 cells (14), a B16F10 variant that has high gp75 surface expression, were cultured in complete DMEM containing 0.2 $\mathrm{mg} / \mathrm{mL}$ Zeocin (Thermo Fisher Scientific, R25001). For the production of IgG, we used the serum-free FreeStyle 293 Expression System (Invitrogen) modified as described previously (70), and further purification was done by protein A (GE Life Sciences), as described by the manufacturer. Cells were cultured at $37^{\circ} \mathrm{C}$ and $5 \% \mathrm{CO}_{2}$.

Expression vector constructs. The variable regions of $\alpha$-EGFR mAb (cetuximab) in a pMA-T vector were purchased from GeneArt (Life Technologies). Linear double-stranded DNA encoding for the variable regions of TA99 ( $\alpha$-gp75), $\alpha$-Fc $\alpha$ RI, and 2 constructs encoding for constant regions of the hIgG1 heavy chains and hIgA2 were purchased from Integrated DNA Technologies. Parental IgG1 antibodies against gp75 and EGFR were of the G1m(f) allotype and contained the point mutation F405L, whereas parental IgG1 $\alpha$-Fc $\alpha$ RI antibodies were of the G1m(a) allotype and contained the K409R mutation in their respective $\mathrm{CH} 3$ domains, enabling the generation of bispecific antibodies as described by Labrijn et al. (43). All linear constructs contained an additional 5 base pairs (GACTG) next to the restriction sites to enable cleavage of incorporated restriction sites. Cloning for the $\alpha$-gp75 antibody has been described previously in Gul et al. (14). Codonoptimized antibodies and isotype variants were generated as described previously $(71,72)$. In short, we designed the codon-optimized variable regions for both heavy and light chains, including $5^{\prime}$-HindIII and $3^{\prime}$-NheI or $5^{\prime}$-HindIII and $3^{\prime}$-XhoI restriction sites, respectively. The constructs were built in the following consecutive order: restriction site, Kozak sequence, and ATG followed by the HAVT20-leader sequence (ref. 71, GeneArt, or Integrated DNA Technologies). The HindIII-NheI or HindIII-XhoI fragments for the codon-optimized heavy and light chains were ligated into vectors encoding for the $\gamma 1$ or $\kappa$-constant regions, respectively. The whole fragments, including the constant regions, were cloned into pcDNA3.1 (Invitrogen) or pEE (Lonza) as HindIII-EcoRI fragments. IgG1 with the F405L mutation [G1m(f) allotype] and the K409R mutation [G1m(a) allotype] were cloned into $\gamma 1$ with 5 '-EcoRV and 3 '-EcoRI restriction sites.

Generation and testing of bispecific IgG1. Bispecific antibodies were generated as previously described (43). In short, the IgG1_F405L and IgG1_K409R antibodies were mixed at equimolar amounts and incubated for 90 minutes at $37^{\circ} \mathrm{C}$ in PBS supplemented with $25 \mathrm{mM}$ 2-MEA (2-mercapto-ethyl-amine hydrochloride, Sigma-Aldrich). After incubation, 2-MEA exchange buffer was removed with Zeba spin desalting columns (7K MWCO, $5 \mathrm{~mL}$, Thermo Fisher Scientific) and left to heterodimerize overnight at $4^{\circ} \mathrm{C}$. Exchange was verified by ELISA utilizing the allotypic differences in the IgG1_F405L and the IgG1_K409R constructs. Antibodies $\alpha$-a (HP6184, 5E7) and $\alpha$-f (HP6185, 5F10) (73) were used, each of which was partially biotinylated according to the EZ-Link Sulfo-NHS-LC-Biotin (Thermo Fisher Scientific) protocol. These antibodies were used in the following sandwich ELISA. First, 96-well plates (Nunc, 439454) were coated with $2 \mu \mathrm{g} / \mathrm{mL} \alpha$-a or $1 \mu \mathrm{g} / \mathrm{mL} \alpha$-f antibodies ( $100 \mu \mathrm{L} /$ well in PBS) overnight at $4^{\circ} \mathrm{C}$. The next day, plates were washed 3 times with PBS-Tween $(0.05 \%[\mathrm{v} / \mathrm{v}]$ Tween 20 [Sigma-Aldrich] in PBS) $(200 \mu \mathrm{L} /$ well) followed by a blocking step with $0.05 \%$ (v/v) milk (skim milk powder, Campina) in PBSTween $(200 \mu \mathrm{L} /$ well) for 1 hour at room temperature. After 3 washes with PBS-Tween, IgG1_F405L [G1m(f) allotype] or IgG1_K409R [G1m(a) allotype] was added at a 2-fold serial dilution from $10 \mu \mathrm{g}$ in PBS-Tween $(100 \mu \mathrm{L} /$ well $)$ and incubated for 1 hour at room temperature. Plates were then washed 3 times with PBS-Tween and $1 \mu \mathrm{g} / \mathrm{mL}$ biotinylated $\alpha$-a or $\alpha$-f antibodies was added in PBS-Tween $(100 \mu \mathrm{L} /$ well) and incubated for 1 hour at room temperature. After 3 washes with PBS-Tween, streptavidin HRP (Sigma-Aldrich) diluted 1:2000 in PBS-Tween was added $(100 \mu \mathrm{L} /$ well $)$ and incubated for 1 hour at room temperature. Plates were washed 3 times with PBS-Tween. After that, TMB substrate was added, which contained $0.1 \mathrm{mg} / \mathrm{mL}$ 3,3',5,5'-tetramethylbenzidine (Merck, 108622), 0.1 M NaAc (Merck, 106267), and $0.003 \%$ (v/v) $\mathrm{H}_{2} \mathrm{O}_{2}$ (Merck, 108597) in MilliQ set to $\mathrm{pH}$ 5.5. After sufficient coloring, $2 \mathrm{M} \mathrm{H}_{2} \mathrm{SO}_{4}$ (Merck, 100731) was added to terminate the reaction.

Fc receptor production. To generate human recombinant Fc $\alpha \mathrm{RI}$ and Fc $\gamma$ R IIIA $158 \mathrm{~V}$, the extracellular domain sequence of these receptors was codon optimized by GeneArt (Life Technologies). 
Subsequently, the sequences were extended with additional C-terminal linker, $10 \times$ His tag and AVI tag, respectively, and flanked by $3^{\prime}$ HindIII and $5^{\prime}$ EcoRV restriction sites. The resulting gene fragments were purchased from Integrated DNA Technologies and were cloned into the pcDNA3.1 expression vector (Thermo Fisher Scientific). The receptors were produced by transfecting HEK293 Freestyle cells (Thermo Fisher Scientific), as previously described (74). Five days after transfection, supernatants were harvested by centrifugation following filtration through a $0.45 \mu \mathrm{m}$ filter (Whatmann, GE Healthcare). Isolation of His-tagged receptors from filtered supernatants was performed by affinity chromatography using a HiTrap column (GE Healthcare) on ÄKTA prime (GE Healthcare) according to the manufacturer's recommended protocol for His isolation. Receptor-containing fractions were subsequently pooled and bufferexchanged into PBS ( $\mathrm{pH}$ 7.4) using Vivaspin concentration tubes with 10K MWCO (GE Healthcare). Final concentrations were determined by Nanodrop 2000c UV/VIS spectrophotometer (Thermo Fisher Scientific). A BirA-500 biotin-protein ligase standard reaction kit (Avidity) was used to examine site-targeted biotinylation on the AVI tag. Degree of biotinylation was verified by ELISA.

SPRi. SPRi measurements were carried out on an IBIS MX96 (IBIS technologies). A g-type streptavidin-coated sensor (SensEye) was spotted using a Continuous Flow Microspotter (Wasatch Microfluidics) with C-terminal biotinylated Fc $\alpha$ RI (30 nM, $10 \mathrm{nM}, 3 \mathrm{nM}$, and $1 \mathrm{nM}$ ). Each run involved 3 subsequent flows. First, either 100 nM TrisomAb $\alpha$-EGFR or $100 \mathrm{nM}$ human IgG1 $\alpha$-EGFR was flowed for 5 minutes. For the second flow, $100 \mathrm{nM} \mathrm{Fc} \gamma \mathrm{RIII}$ 158V or 100 $\mathrm{nM}$ FcaRI was flowed for 5 minutes. The last flow consisted of $1 \times$ $10^{6}$ cells $/ \mathrm{mL}$ of either A431, HCT116, or SW620 cells or system buffer $(0.1 \%$ [w/v] BSA in PBS, pH 7.4) for 20 minutes of sedimentation and washing with increasing flow speeds. The affinity of TrisomAb $\alpha$-EGFR, human IgG1 $\alpha$-EGFR, and human IgG1 $\alpha$-Fc $\alpha$ RI toward Fc $\alpha$ RI and human Fc $\gamma$ RIIIa $158 \mathrm{~V}$ protein was determined using a previously described method (75). In short, biotinylated Fc $\alpha$ RI and Fc $\gamma$ RIIIa $158 \mathrm{~V}$ were spotted in triplicate on a g-type streptavidin sensor (SensEye) in 4 concentrations ranging from $30 \mathrm{nM}$ to $1 \mathrm{nM}$. Trisom$\mathrm{Ab} \alpha$-EGFR, human IgG1 $\alpha$-EGFR, and human IgG1 $\alpha$-Fc $\alpha$ RI (custom $\mathrm{mAbs}$, see Expression vector constructs) were flowed over the sensor in 12 concentrations ranging from $0.12 \mathrm{nM}$ to $250 \mathrm{nM}$. Affinities were calculated as described by Dekkers et al. (75).

ADCC assay. NK cells were isolated according to the manufacturer's protocol (Miltenyi Biotec, 130-092-657). PMNs were isolated as previously described (76). Enriched neutrophils were isolated using the EasySep human neutrophil isolation kit (Stemcell Technologies, 17957). Killing assays were performed at an effector to target ratio of 5:1 for NK cells and 50:1 for PMNs and enriched neutrophils using A431 or B16F10gp75 as target cells. Cell survival was determined using the CellTiter-Blue (CTB) Cell Viability Assay (Promega, G8080). Target cells (8000 cells/well in a flat-bottom 96-well plate) were cultured for 24 hours. The next day, culture medium was refreshed with $50 \mu \mathrm{L} /$ well complete RPMI 1640 in the presence of antibodies $(10 \mu \mathrm{L} /$ well, 11 times the test concentration in RPMI 1640 medium) in various concentrations, i.e., $0.1,1$, and $10 \mu \mathrm{g} / \mathrm{mL}$. Next, $50 \mu \mathrm{L} /$ well freshly isolated PMNs or enriched neutrophils $\left(8 \times 10^{6}\right.$ cells $/ \mathrm{mL}$ in RPMI 1640 medium $)$ or NK cells $\left(8 \times 10^{5}\right.$ cells $/ \mathrm{mL}$ in RPMI 1640 medium) were cocultured with the target cells for either 4 or 24 hours at $37^{\circ} \mathrm{C}$ and $5 \% \mathrm{CO}_{2}$.
Target cells alone, effector cells alone, RPMI 1640 medium alone, and no antibody were used as controls. Flat-bottom 96-well plates were washed twice with $200 \mu \mathrm{L} /$ well PBS, blotted dry, and incubated with $100 \mu \mathrm{L} /$ well CTB reagent (CTB stock concentration was diluted 1:6 in complete RPMI 1640) for 1 hour at $37^{\circ} \mathrm{C}$ and $5 \% \mathrm{CO}_{2}$. Incubation plates were shaken at $400 \mathrm{rpm}$ for 5 minutes and fluorescence of formed resorufin, proportional to the amount of living cells in the wells, was recorded by a FLUOstar Galaxy Microplate Reader (MXT Lab Systems). Resorufin was excited using a 560/10 $\mathrm{nm}$ excitation filter and emission was acquired using a 590/12 nm filter. Gain of the detector was set to 115 (gain 0-255). The percentage of cellular cytotoxicity was calculated in Microsoft Excel using the equation $\%$ cytotoxicity $=(1-$ [experimental value - medium control] $/$ [no antibody - medium control]) $\times 100$. Data were plotted and analyzed using GraphPad Prism 7.0.

$A D C P$ assay. Monocytes were isolated as previously described (76) and cultured for 6 days in the presence of $50 \mathrm{ng} / \mathrm{mL} \mathrm{M-CSF}$ to generate macrophages. Macrophages were counted, labeled with DiO (Molecular Probes, D275), and seeded in 24-well plates $(1.5 \times$ $10^{5}$ cells/well). After 48 hours, culture medium was refreshed with $250 \mu \mathrm{L} /$ well complete RPMI 1640 in the presence of therapeutic antibodies $(125 \mu \mathrm{L} /$ well, 4 times the test concentration in RPMI 1640 medium) in various concentrations, i.e., 0.001, 0.01, 0.1, 1, and 10 $\mu \mathrm{g} / \mathrm{mL}$. Next, A431 or B16F10gp75 cells labeled with cell proliferation dye eFluor 450 (eBioscience, 65-0842-85) were added as target cells $(125 \mu \mathrm{L} /$ well containing approximately 240,000 cells $/ \mathrm{mL})$ to obtain an effector to target ratio of 5:1. Tumor cells were cocultured with macrophages for 4 hours at $37^{\circ} \mathrm{C}$ and $5 \% \mathrm{CO}_{2}$. Cells were washed with PBS and harvested by trypsin treatment $(250 \mu \mathrm{L} /$ well, 15 minutes incubation at $37^{\circ} \mathrm{C}$ and $5 \% \mathrm{CO}_{2}$ ) followed by cell scraping and fixing with $1 \%$ formaldehyde in PBS. Samples were analyzed with flow cytometry (BD LSRFortessa) and FlowJo software. Percentage phagocytic events was quantified using the equation $\%$ phagocytosis $=(1-[\%$ tumor cells in experiment $] /[\%$ tumor cells without antibody]) $\times 100$.

ELISA. Lactoferrin and IL-8 release in supernatants of neutrophil-mediated killing assays were determined using matched antibody pairs. Rabbit $\alpha$-human lactoferrin (Sigma-Aldrich, L3262), alkaline phosphatase-labeled rabbit $\alpha$-human lactoferrin-AF (MP Biomedicals, 675581), and p-nitrophenyl phosphate (SigmaAldrich, N1891) as chromogenic substrate were used for lactoferrin detection as previously described (77). IL-8 in supernatants was measured using the human IL-8 Ready-SET-Go ELISA Set (Invitrogen, 50-246-341) according to the manufacturer's protocol. Mouse $\alpha$-human IL-4 (Thermo Fisher Scientific, AHC0642) and biotinmouse $\alpha$-human IL-4 (Thermo Fisher Scientific, AHC0749) were used as primary and secondary antibody, respectively, followed by addition of streptavidin-HRP (Thermo Fisher Scientific, SNN2004) for IL-4 detection. IL-13 and histamine were detected using the human IL3 ELISA kit (Invitrogen, 88-7439-88) and the human histamine ELISA kit (Mybiosource.com, MBS733349), respectively. FAE of BsAbs was verified using an ELISA with matched antibody pairs against G1m(a) and G1m(f) allotypes (Sigma-Aldrich, I5385).

Live-cell imaging. First, $4 \times 10^{5} \mathrm{~A} 431$ Lifeact $\mathrm{mScarlet}$ cells were cultured on round cover glasses (Harvard Apparatus, 1217N82, \#1.5 thickness, $25 \mathrm{~mm}$ ) coated with collagen from calf skin (SigmaAldrich, C9791) for 24 hours. Tumor colonies were labeled with 
the cell-permeant dye calcein AM (Thermo Fisher Scientific, C3100MP), or a caspase-3/7 sensor (Thermo Fisher Scientific, C10723) to follow the induction of antibody-mediated apoptosis according to the manufacturer's protocols. Labeled tumor cells on cover glasses were transferred to an Attofluor Cell Chamber for microscopy (Thermo Fisher Scientific, A7816). Next, $2 \times 10^{6} \mathrm{NK}$ cells or neutrophils were labeled with cell proliferation dye eFluor 670 (eBioscience, 65-0840-85) and therapeutic antibodies $(2 \mu \mathrm{g} /$ $\mathrm{mL}$ ) were added. Neutrophil-tumor cell and NK cell-tumor cell interactions were recorded for 40 and 180 minutes, respectively, by a Nikon Eclipse Ti microscope equipped with hybrid photo detectors and resonant scanner for fast image acquisition. The microscope contained an Okolab cage incubator as well as a perfect focus system to allow stable time-lapse imaging with automatic focus correction under controlled humidity, $\mathrm{CO}_{2}$, and $\mathrm{O}_{2}$ conditions. After acquisition, neutrophil and tumor cell behavior, i.e., number of interactions, distance to tumor, longest contact, and tumor area, were analyzed offline using tracking software (Imaris, bitplane) and a custom-written Xtension for Imaris named Kiss and Run Analysis. Representative movies and images were generated by Fiji and Imaris software.

Western blot. To prepare crude cell lysates, cells were washed and resuspended in RPMI 1640 without serum. Subsequently, cells were incubated with a human IgG1 antibody against FcaRI (positive control, $\alpha$-Fc $\alpha$ RI parental of TrisomAb), a human IgG1 antibody against EGFR (cetuximab), or TrisomAb $\alpha$-EGFR (custom mAbs, see Expression vector constructs) for 30 minutes on ice. After washing, cells were crosslinked using $\mathrm{F}\left(\mathrm{ab}^{\prime}\right) 2$ goat $\alpha$-human fragments (Invitrogen, A24464). Cells were incubated at $37^{\circ} \mathrm{C}$ for $0,1,2,3,5,10$, or 20 minutes, after which cells were boiled for 5 minutes at $95^{\circ} \mathrm{C}$ in preheated $2 \times$ Laemmli buffer containing 8\% 2-mercaptoethanol. Samples were thoroughly vortexed, spun down, and kept at $-20^{\circ} \mathrm{C}$ until usage. For Western blotting, samples were loaded on $10 \%$ polyacrylamide gels according to standard procedures. Membranes were incubated overnight with mouse $\alpha$-phosphotyrosine (4G10, EMD Millipore) and rabbit $\alpha$-GAPDH (14C10, Cell Signaling Technology) antibodies. As secondary antibodies, goat $\alpha$-mouse-IRDye $800 \mathrm{CW}$ and goat $\alpha$-rabbit-IRDye 680RD antibodies (Odyssey, LI-COR) were used. Membranes were analyzed using an Odyssey Classic Imager (LI-COR). Pixel intensity of bands at 40 and $70 \mathrm{kDa}$ was determined with Image Studio Lite (LI-COR). GAPDH quantification was used to correct for protein loading in total lysates.

Flow cytometry. A431 and B16F10gp75 cells and PMNs were incubated with different concentrations of TrisomAb $\alpha$-EGFR, TrisomAb $\alpha$-gp75, hIgG1 $\alpha$-Fc $\alpha$ RI, hIgG1 isotype control, and hIgG1 $\alpha$-EGFR (cetuximab) (custom mAbs, see Expression vector constructs) for 45 minutes on ice. After washing, primary antibody was detected by incubation with PE-conjugated goat $\alpha$-human IgG mAb (1:50). Cells were analyzed with flow cytometry (BD LSRFortessa) and FlowJo software.

Neutrophil chemotaxis assay. Supernatants from neutrophilmediated killing experiments were harvested, and migration of freshly isolated neutrophils toward supernatants was determined with neutrophil chemotaxis assays as previously described (78). In short, PMNs were fluorescently labeled for 30 minutes at $37^{\circ} \mathrm{C}$ with calcein AM (Thermo Fisher Scientific, C3100MP). After incubation, cells were washed twice and carefully resuspended in RPMI 1640. To measure chemotaxis (directed migration), bottom wells of blind well chambers were filled with supernatants from neutrophil-mediated killing experiments or purified $1 \times 10^{-7} \mathrm{M}$ LTB4 (Sigma-Aldrich, $1027 \mathrm{M}$ ) as positive control. Next, $5 \times 10^{4}$ calcein AM-labeled neutrophils were added to the top wells. After incubation for 40 minutes at $37^{\circ} \mathrm{C}$, cells that had migrated toward the lower compartments were quantified in black 96-well plates (FLUOTRAC200) with a fluorimeter (FLUOstar/POLARstar) at $480 \mathrm{~nm}$ excitation, $520 \mathrm{~nm}$ emission.

In vivo serum half-life of TrisomA $b$. Human IgG1 $\alpha$-gp75, hIgA2 $\alpha$-gp75, or TrisomAb $\alpha$-gp75 (50 $\mu \mathrm{g}$ ) (custom mAbs, see Expression vector constructs) was i.p. injected into C57BL/6 LysM-EGFP mice (3 mice per time point). Blood was collected by cardiac puncture blood sampling (terminal) after the indicated time points, and the antibody concentration in the serum was determined by ELISA. Half-life was calculated using the formula $t_{1 / 2}=\Delta t / 2 \log (C 1 / C 2)$.

Subcutaneous syngeneic tumor model. Human FcaRI transgenic $\times$ LysM-EGFP mice and Fc $\alpha$ RI transgene-negative littermates of LysM C57BL/6 mice were s.c. inoculated with $1 \times 10^{4}$ B16F10gp75 tumor cells. Antibodies (100 $\mu \mathrm{g}$ in $200 \mu \mathrm{L} \mathrm{PBS})$ were i.p. injected on days 0, 1 , and 2. Tumor growth was monitored and scored for 16 days using caliper size measurements. Tumor volume (in $\mathrm{mm}^{3}$ ) was calculated using the equation $V=\pi \times(W \times L \times 0.5)^{3} / 6$, where $V$ is tumor volume, $W$ is tumor width in $\mathrm{mm}$, and $L$ is tumor length in $\mathrm{mm}$. For survival experiments, mice were terminated when tumors reached the humane endpoint (main criterion: tumor volume $>1000 \mathrm{~mm}^{3}$ ).

Immune cell depletions. Neutrophils were depleted by i.p. injection with $400 \mu \mathrm{g} \alpha$-Ly6G/Ly6C (Gr-1) mAb (clone RB6-8C5, BioXCell) and NK cells were depleted by i.p. injection of $200 \mu \mathrm{g} \alpha-\mathrm{NK} 1.1 \mathrm{mAb}$ (clone PK136) in $200 \mu \mathrm{L}$ PBS given 2 times a week beginning 1 day before initiation of TrisomAb therapy and tumor inoculation. No depletion groups received isotype control mAbs (clone 2A3, BioXCell). Depletion was verified by flow cytometry in blood 3 days after the first injection with depleting antibodies (Supplemental Figure 8, A and B). Monocyte and macrophage populations were depleted by i.p. injection of 300 $\mu \mathrm{g} \alpha$-CSF1R (clone AFS98, BioXCell) in $200 \mu \mathrm{L}$ PBS given every other day beginning 5 days before initiation of TrisomAb therapy and tumor inoculation. Macrophage depletion was confirmed by flow cytometry in blood and spleen 5 days after injection of $\alpha$-CSF1R (Supplemental Figure 8, C and D). Samples were stained for CD45 and CD11b. NK cells were identified by NKp46 staining; splenic macrophages were identified by $\mathrm{F} 4 / 80$ staining.

Statistics. Statistical comparison between experimental groups was performed using 2-way ANOVA tests corrected for multiple comparisons by Tukey's multiple-comparison test with a single pooled variance. Statistical comparisons between experimental groups for SPRi data were performed by multiple $t$ tests. A 2-tailed $P$ value of less than 0.05 was considered significant. Unless otherwise stated, representative experiments out of at least 3 independent experiments are shown. Survival curves were analyzed using the logrank (Mantel-Cox) test.

Study approval. All animal experiments were approved by the Animal Ethical Committee of the VU University and VU Medical Center (AVD1140020173844). All experiments were performed according to institutional and national guidelines. Human PMNs, NK cells, and monocytes were isolated from whole blood derived from anonymized, healthy adult volunteers who signed an informed consent under the rules and legislation in place within the Netherlands and maintained by 
the VU Medical Center Medical Ethical Committee (METC). The use of colorectal cancer patient samples (METC VU Medical Center: 2016.271) was approved by the VU Medical Center METC in accordance with the Declaration of Helsinki and guidelines for Good Clinical Practice.

\section{Author contributions}

$\mathrm{NH}$ designed, performed, and analyzed the imaging and in vivo experiments and wrote the manuscript. ART, SLT, and AEHB produced TrisomAb and performed the experiments verifying the format of TrisomAb. Western blotting experiments were done by $\mathrm{MHH}$ and PJW. CWT and MG performed the ADCC, ADCP, chemotaxis, and ELISA experiments. TH collected the patient samples. MDD helped with imaging experiments. DYG, MB, and JEB advised on the design of ADCC, Western blotting, and in vivo experiments. TR and TV provided the IgA $\alpha$-EGFR antibody. GV supervised the study and cowrote the paper. MVE supervised and funded the study and cowrote the manuscript.

\section{Acknowledgments}

This work is supported by Worldwide Cancer Research (grant 15-1240) and Cancer Center Amsterdam (to MVE). RBL-hCD89 cells and LysM-EGFP mice were a gift from Renato C. Monteiro (Faculté de Médecine, Université Paris Diderot) and Thomas Graf (Centre for Genomic Regulation), respectively. Lifeact mScarlet was a gift from Jaap D. van Buul (Sanquin Research and Landsteiner Laboratory, Amsterdam UMC), Joachim Goedhart and TWJ Gadella Jr. (Swammerdam Institute for Life Sciences, University of Amsterdam, Amsterdam, the Netherlands). We would like to thank Katarzyna Olesek for technical assistance.

Address correspondence to: Marjolein van Egmond, VU University Medical Center, Department of Surgery, ZH 7F018, De Boelelaan 1117, 1081 HV Amsterdam, The Netherlands. Phone: 31.20.444.4536; Fax: 31.20.444.8081; Email: m.vanegmond@ amsterdamumc.nl.
1. Couzin-Frankel J. Breakthrough of the year 2013. Cancer immunotherapy. Science. 2013;342(6165):1432-1433.

2. Weiner GJ. Building better monoclonal antibody-based therapeutics. Nat Rev Cancer. 2015;15(6):361-370.

3. Sliwkowski MX, Mellman I. Antibody therapeutics in cancer. Science. 2013;341(6151):1192-1198.

4. Ferris RL, et al. Rationale for combination of therapeutic antibodies targeting tumor cells and immune checkpoint receptors: harnessing innate and adaptive immunity through IgG1 isotype immune effector stimulation. Cancer Treat Rev. 2018;63:48-60.

5. Chanan-Khan A, et al. Ibrutinib combined with bendamustine and rituximab compared with placebo, bendamustine, and rituximab for previously treated chronic lymphocytic leukaemia or small lymphocytic lymphoma (HELIOS): a randomised, double-blind, phase 3 study. Lancet Oncol. 2016;17(2):200-211.

6. Marshall MJE, et al. Therapeutic antibodies: what have we learnt from targeting CD20 and where are we going? Front Immunol. 2017;8:1245.

7. Byeon HK, et al. Beyond EGFR inhibition: multilateral combat strategies to stop the progression of head and neck cancer. Exp Mol Med. 2019;51(1):1-14.

8. Scott AM, et al. Antibody therapy of cancer. Nat Rev Cancer. 2012;12(4):278-287.

9. Smyth MJ, et al. Combination cancer immunotherapies tailored to the tumour microenvironment. Nat Rev Clin Oncol. 2016;13(3):143-158.

10. Golay J, Introna M. Mechanism of action of therapeutic monoclonal antibodies: promises and pitfalls of in vitro and in vivo assays. Arch Biochem Biophys. 2012;526(2):146-153.

11. Reis ES, et al. Complement in cancer: untangling an intricate relationship. Nat Rev Immunol. 2018;18(1):5-18.

12. Guillerey C, et al. Targeting natural killer cells in cancer immunotherapy. Nat Immunol. 2016;17(9):1025-1036.

13. Chiossone L, et al. Natural killer cells and other innate lymphoid cells in cancer. Nat Rev Immunol. 2018;18(11):671-688.
14. Gül N, et al. Macrophages eliminate circulating tumor cells after monoclonal antibody therapy. J Clin Invest. 2014;124(2):812-823.

15. Braster R, et al. Myeloid cells as effector cells for monoclonal antibody therapy of cancer. Methods. 2014;65(1):28-37.

16. Vargas FA, et al. Fc effector function contributes to the activity of human anti-CTLA-4 antibodies. Cancer Cell. 2018;33(4):649-663.

17. Nimmerjahn F, et al. Divergent immunoglobulin g subclass activity through selective Fc receptor binding. Science. 2005;310(5753):1510-1512.

18. Park SG, et al. The therapeutic effect of anti-HER2/ neu antibody depends on both innate and adaptive immunity. Cancer Cell. 2010;18(2):160-170.

19. Kaifu T, Nakamura A. Polymorphisms of immunoglobulin receptors and the effects on clinical outcome in cancer immunotherapy and other immune diseases: a general review. Int Immunol. 2017;29(7):319-325

20. Wang B, et al. Fc $\gamma$ R polymorphisms and clinical outcome in colorectal cancer patients receiving passive or active antibody treatment. Int JOncol. 2010;37(6):1599-1606.

21. Hatjiharissi E, et al. Increased natural killer cell expression of CD16, augmented binding and ADCC activity to rituximab among individuals expressing the Fc \{gamma\}RIIIa-158 V/V and V/F polymorphism. Blood. 2012;110(7):2561-2564.

22. Kasper S, et al. Oncogenic RAS simultaneously protects against anti-EGFR antibody-dependent cellular cytotoxicity and EGFR signaling blockade. Oncogene. 2013;32(23):2873-2881.

23. Del Re M, et al. Implications of KRAS mutations in acquired resistance to treatment in NSCLC. Oncotarget. 2017;9(5):6630-6643.

24 . Di Carlo E, et al. The intriguing role of polymorphonuclear neutrophils in antitumor reactions. Blood. 2001;97(2):339-345.

25. van Egmond M, Bakema JE. Neutrophils as effector cells for antibody-based immunotherapy of cancer. Semin Cancer Biol. 2013;23(3):190-199.

26. Stockmeyer B, et al. Mechanisms of G-CSFor GM-CSF-stimulated tumor cell killing by Fc receptor-directed bispecific antibodies. J Immunol Methods. 2001;248(1-2):103-111.
27. Heemskerk N, van Egmond M. Monoclonal antibody-mediated killing of tumour cells by neutrophils. Eur JClin Invest. 2018;48(suppl 2):e12962.

28. Bakema JE, et al. Targeting Fc RI on polymorphonuclear cells induces tumor cell killing through autophagy. JImmunol. 2011;187(2):726-732.

29. Carmi Y, et al. Allogeneic IgG combined with dendritic cell stimuli induce antitumour T-cell immunity. Nature. 2015;521(7550):99-104.

30. DiLillo DJ, Ravetch JV. Differential Fc-receptor engagement drives an anti-tumor vaccinal effect. Cell. 2015;161(5):1035-1045.

31. Zhu EF, et al. Synergistic innate and adaptive immune response to combination immunotherapy with anti-tumor antigen antibodies and extended serum half-life IL-2. Cancer Cell. 2015;27(4):489-501.

32. Moynihan KD, et al. Eradication of large established tumors in mice by combination immunotherapy that engages innate and adaptive immune responses. Nat Med. 2016;22(12):1402-1410.

33. van Gisbergen KPJM, et al. Neutrophils mediate immune modulation of dendritic cells through glycosylation-dependent interactions between Mac-1 and DC-SIGN. J Exp Med. 2005;201(8):1281-1292.

34. Albanesi M, et al. Neutrophils mediate antibody-induced antitumor effects in mice. Blood. 2013;122(18):3160-3164.

35. Matlung HL, et al. Neutrophils kill antibodyopsonized cancer cells by trogoptosis. Cell Rep. 2018;23(13):3946-3959.e6.

36. Dechant M, et al. Effector mechanisms of recombinant IgA antibodies against epidermal growth factor receptor. J Immunol. 2007;179(5):2936-2943.

37. Otten MA, et al. Enhanced Fc $\alpha$ RI-mediated neutrophil migration toward tumour colonies in the presence of endothelial cells. Eur JImmunol. 2012;42(7):1815-1821.

38. Otten MA, et al. Immature neutrophils mediate tumor cell killing via IgA but not IgG Fc receptors. JImmunol. 2005;174(9):5472-5480.

39. Boross P, et al. IgAEGFR antibodies mediate tumour killing in vivo. EMBO Mol Med 2013;5(8):1213-1226. 
40. Brandsma AM, et al. Potent Fc receptor signaling by IgA leads to superior killing of cancer cells by neutrophils compared with IgG. Front Immunol. 2019;10:704.

41. Brandsma AM, et al. Simultaneous targeting of Fc $\alpha$ Rs and Fc $\gamma$ RI enhances tumor cell killing. Cancer Immunol Res. 2015;3(12):1316-1324.

42. Huls G, et al. Antitumor immune effector mechanisms recruited by phage display-derived fully human IgG1 and IgA1 monoclonal antibodies. Cancer Res. 1999;59(22):5778-5784.

43. Labrijn AF, et al. Efficient generation of stable bispecific IgG1 by controlled Fab-arm exchange. Proc Natl Acad Sci U S A. 2013;110(13):5145-5150.

44. Launay P, et al. Alternative endocytic pathway for immunoglobulin A Fc receptors (CD89) depends on the lack of FcRgamma association and protects against degradation of bound ligand. J Biol Chem. 1999;274(11):7216-7225.

45. Coffelt SB, et al. IL-17-producing $\gamma \delta \mathrm{T}$ cells and neutrophils conspire to promote breast cancer metastasis. Nature. 2015;522(7556):345-348.

46. van Egmond M, et al. Human immunoglobulin A receptor (FcalphaRI, CD89) function in transgenic mice requires both $\mathrm{FcR}$ gamma chain and CR3 (CD11b/CD18). Blood.1999;93(12):4387-4394.

47. Rouwendal GJA, et al. A comparison of anti-HER2 IgA and IgG1 in vivo efficacy is facilitated by high $\mathrm{N}$-glycan sialylation of the IgA. MAbs. 2016;8(1):74-86.

48. Morell A, et al. Metabolic properties of human IgA subclasses. Clin Exp Immunol. 1973;13(4):521-528.

49. Durgeau A, et al. Recent advances in targeting CD8 T-cell immunity for more effective cancer immunotherapy. Front Immunol. 2018;9:14

50. Turaj AH, et al. Antibody tumor targeting is enhanced by CD27 agonists through myeloid recruitment. Cancer Cell. 2017;32(6):777-791.e6.

51 . Woo SR, et al. Innate immune recognition of cancer. Annu Rev Immunol. 2015;33:445-474.

52. Repp R, et al. Neutrophils express the high affinity receptor for IgG (Fc gamma RI, CD64) after in vivo application of recombinant human granulocyte colony-stimulating factor. Blood. 1991;78(4):885-889.

53. Valerius $\mathrm{T}$, et al. Involvement of the highaffinity receptor for IgG (Fc gamma RI; CD64) in enhanced tumor cell cytotoxicity of neutrophils during granulocyte colony-stimulating factor therapy. Blood. 1993;82(3):931-939.
54. Fury MG, et al. A phase-I trial of the epidermal growth factor receptor directed bispecific antibody MDX-447 without and with recombinant human granulocyte-colony stimulating factor in patients with advanced solid tumors. Cancer Immunol Immunother. 2008;57(2):155-163.

55. James ND, et al. A phase II study of the bispecific antibody MDX-H210 (anti-HER2 x CD64) with GM-CSF in HER2 ${ }^{+}$advanced prostate cancer. Br J Cancer. 2001;85(2):152-156.

56. Valerius T, et al. FcalphaRI (CD89) as a novel trigger molecule for bispecific antibody therapy. Blood.1997;90(11):4485-4492.

57. Deo YM, et al. Bispecific molecules directed to the Fc receptor for IgA (Fc alpha RI, CD89) and tumor antigens efficiently promote cell-mediated cytotoxicity of tumor targets in whole blood. J Immunol. 1998;160(4):1677-1686.

58. Guettinger $Y$, et al. A recombinant bispecific single-chain fragment variable specific for HLA class II and Fc alpha RI (CD89) recruits polymorphonuclear neutrophils for efficient lysis of malignant B lymphoid cells. JImmunol. 2010;184(3):1210-1217.

59. Borrok JM, et al. Enhancement of antibodydependent cell-mediated cytotoxicity by endowing IgG with Fc $\alpha$ RI (CD89) binding. MAbs. 2015;7(4):743-751.

60. Kelton W, et al. IgGA: a 'cross-isotype' engineered human Fc antibody domain that displays both IgG-like and IgA-like effector functions. Chem Biol. 2014;21(12):1603-1609.

61. Li B, et al. Simultaneous exposure to Fc $\gamma$ R and $\mathrm{Fc} \alpha \mathrm{R}$ on monocytes and macrophages enhances antitumor activity in vivo. Oncotarget. 2017;8(24):39356-39366.

62. Otten MA, et al. Immature neutrophils mediate tumor cell killing via IgA but not IgG Fc receptors. JImmunol. 2005;174(9):5472-5480.

63. Lämmermann T, et al. Neutrophil swarms require LTB4 and integrins at sites of cell death in vivo. Nature. 2013;498(7454):371-375.

64. van der Steen L, et al. Immunoglobulin A: Fc $\alpha$ RI interactions induce neutrophil migration through release of leukotriene B4. Gastroenterology. 2009;137(6):2018-2029.

65. van Spriel AB, et al. Mac-1 (CD11b / CD18) is essential for Fc receptor-mediated neutrophil cytotoxicity and immunologic synapse formation. Blood. 2001;97(8):2478-2486.

66. Treffers LW, et al. IgA-mediated killing of tumor cells by neutrophils is enhanced by CD47-SIRPA checkpoint inhibition. Cancer Immunol Res. 2020;8(1):120-130

67. van Montfoort N, et al. Antigen storage compartments in mature dendritic cells facilitate prolonged cytotoxic T lymphocyte cross-priming capacity. Proc Natl Acad Sci US A. 2009;106(16):6730-6735.

68. Clatworthy MR, et al. Immune complexes stimulate CCR7-dependent dendritic cell migration to lymph nodes. Nat Med. 2014;20(12):1458-1463.

69. Faust N, et al. Insertion of enhanced green fluorescent protein into the lysozyme gene creates mice with green fluorescent granulocytes and macrophages. Blood. 2000;96(2):719-726

70. Dekkers G, et al. Multi-level glyco-engineering techniques to generate $\operatorname{IgG}$ with defined $\mathrm{Fc}$ glycans. Sci Rep. 2016;6:36964.

71. Vidarsson G, et al. Activity of human IgG and IgA subclasses in immune defense against Neisseria meningitidis serogroup B. JImmunol. 2001;166(10):6250-6256.

72. Dekkers G, et al. Decoding the human immunoglobulin G-glycan repertoire reveals a spectrum of Fc-receptor- and complement-mediatedeffector activities. Front Immunol. 2017;8:877.

73. Jefferis R, et al. Evaluation of monoclonal antibodies having specificity for human IgG subclasses: results of the 2nd IUIS/WHO collaborative study. Immunol Lett. 1992;31(2):143-168.

74. Vink T, et al. A simple, robust and highly efficien transient expression system for producing antibodies. Methods. 2014;65(1):5-10.

75. Dekkers G, et al. Affinity of human IgG subclasses to mouse Fc gamma receptors. MAbs. 2017;9(5):767-773.

76. Heemskerk N, et al. F-actin-rich contractile endothelial pores prevent vascular leakage during leukocyte diapedesis through local RhoA signalling. Nat Commun. 2016;7:10493.

77. Aleyd E, et al. IgA complexes in plasma and synovial fluid of patients with rheumatoid arthritis induce neutrophil extracellular traps via FcaRI. Jimmunol. 2016;197(12):4552-4559.

78. Frevert CW, et al. Rapid fluorescence-based measurement of neutrophil migration in vitro. J Immunol Methods. 1998;213(1):41-52.

79. Matsumiya S, et al. Structural comparison of fucosylated and nonfucosylated Fc fragments of human immunoglobulin G1. J Mol Biol. 2007;368(3):767-779. 